\title{
Vortex dislocations in wake-type flow induced by spanwise disturbances
}

\author{
Guo Can Ling ${ }^{\text {a) }}$ and Hong Liang Zhao \\ The State Key Laboratory of Nonlinear Mechanics, Institute of Mechanics, Chinese Academy of Sciences, \\ Beijing 100190, China
}

(Received 7 November 2008; accepted 22 June 2009; published online 31 July 2009)

\begin{abstract}
Vortex dislocations in wake-type flow induced by three types of spanwise disturbances superimposed on an upstream velocity profile are investigated by direct numerical simulations. Three distinct modes of vortex dislocations and flow transitions have been found. A local spanwise exponential decay disturbance leads to the appearance of a twisted chainlike mode of vortex dislocation. A stepped spanwise disturbance causes a streamwise periodic spotlike mode of vortex dislocation. A spanwise sinusoidal wavy disturbance with a moderate waviness causes a strong unsteadiness of wake behavior. This unsteadiness starts with a systematic periodic mode of vortex dislocation in the spanwise direction followed by the spanwise vortex shedding suppressed completely with increased time and the near wake becoming a steady shear flow. Characteristics of these modes of vortex dislocation and complex vortex linkages over the dislocation, as well as the corresponding dynamic processes related to the appearance of dislocations, are described by examining the variations of vortex lines and vorticity distribution. The nature of the vortex dislocation is demonstrated by the substantial vorticity modification of the spanwise vortex from the original spanwise direction to streamwise and vertical directions, accompanied by the appearance of noticeable vortex branching and complex vortex linking, all of which are produced at the locations with the biggest phase difference or with a frequency discontinuity between shedding cells. The effect of vortex dislocation on flow transition, either to an unsteady irregular vortex flow or suppression of the Kármán vortex shedding making the wake flow steady state, is analyzed. Distinct similarities are found in the mechanism and main flow phenomena between the present numerical results obtained in wake-type flows and the experimental-numerical results of cylinder wakes reported in previous studies. (C) 2009 American Institute of Physics. [DOI: 10.1063/1.3192652]
\end{abstract}

\section{INTRODUCTION}

A number of experimental investigations have shown that in some vortex dominated flows, such as the cylinder wake, mixing layer, and some other nonlinear waves, the flow transition involves the appearance of large-scale vortex dislocations or topological defects of vortex patterns with a complex three-dimensional configuration. Studies of the wake three dimensionalities caused by the spanwise variation either in velocity or diameter have also shown that the vortex dislocation can be forced to occur. Depending on these threedimensional flow conditions, vortex dislocations exhibit various modes and complex topology of streamwise vortex lines. Vortex dislocations are generated between spanwise vortex shedding cells with differences in phase or frequency. They play an important role in the flow transition and are considered to be a new kind of mechanism for the transition. Detailed presentations of them can be found in experimental studies of Williamson ${ }^{1,2}$ and Lewis and Gharib. ${ }^{3}$ A number of other authors also have reported results of interesting observations and investigations on the vortex dislocation appearing in wakes and mixing layer. For details the reader is referred to the works of Bearman, ${ }^{4}$ Eisenlohr and

\footnotetext{
${ }^{\text {a) }}$ Author to whom correspondence should be addressed. Telephone: 0086010-82543916. Fax: 0086-010-82543977. Electronic mail: linggc@imech.ac.cn.
}

Eckelemann, ${ }^{5}$ Gerrard, ${ }^{6}$ Papangelou, ${ }^{7}$ Darekar and Sherwin, ${ }^{8}$ Dallard and Browand, ${ }^{9}$ among others.

It is clear that the majority of previous experimental studies have been devoted to exploration of the generation of vortex dislocation and its effect on wake development behind the cylinder with geometric variations. However there is no great deal of investigation on vortex dislocation in shear flow, such as wake-type shear flow with spanwise nonuniformities. In this regard, physical understanding of the precise mechanism of the formation of vortex dislocation and the details of vortex linkages over the dislocation are far from complete.

In recent numerical studies, by means of direct numerical simulation (DNS) Braza et al. ${ }^{10}$ investigated the natural vortex dislocation in three-dimensional wake transition. Darekar and Sherwin ${ }^{8}$ studied the flow past a square-section cylinder with a wavy stagnation face. Ling and Zhao ${ }^{11}$ and Ling and $\mathrm{Niu}^{12}$ performed some numerical studies for exploration of vortex dislocation caused by a local disturbance in a wake-type flow. However for qualitative and quantitative understanding of dynamical behavior of vortex in various practical wake flows and many basic problems, which require parametric investigations, such DNS computations could still become prohibitively expensive. A numerical efficient way to study the wake vortex dynamics was proposed by Triantafyllou and Karniadakis. ${ }^{13}$ They demonstrated that the vortex wake of bluff bodies, particularly a Kármán vortex 
street, could be reproduced knowing the time-averaged flow at a specific location behind a cylinder, where such flow was the most unstable as analyzed from linear instability theory. That gives strong credence to almost universal practice of simply disregarding the cylinder body in wake stability analysis. Further it opens new possibilities by studying the evolution of the time-averaged flow with spanwise variation to investigate the nonlinear interaction of vortices produced in the wake flows.

The interest of the present work is in the study of the fundamental features and the dynamics of vortex dislocation appearing in wake flows by the DNS approach. Of particular importance is to understand the influence of different types of spanwise nonuniform disturbance imposed on the twodimensional streamwise upstream flow on the vortex dislocation and wake development. Following the concept of computational reducibility by Triantafyllou and Karniadakis ${ }^{13}$ mentioned above, this study tries to simplify the complex vortex shedding phenomena induced by a real cylinder with spanwise diameter variation, using a wake-type shear flow profile with different spanwise variations in streamwise velocity defects, and to gain some basic knowledge on the mechanism of vortex dislocations appearing in the real flows. According to linear instability analysis, with different time-averaged streamwise upstream velocity profiles obtained from previous experiments, vortex streets can be obtained with different shedding frequencies along the span of the flow. By studying the evolution of this kind of flow, we expected to obtain an understanding of the phenomena of vortex dislocation without using a large amount of computation resources. It was also suggested that the results of this study would not be the same as those given by the experiments but that they would mimic, in some aspects, the vortex dislocations produced by real cylinder with diameter variation. It was presumed that the present results will be helpful to well understand the complexity and intrinsic mechanism on vortex dislocation occurring in real cylinder wakes. On the other hand, since the evolution of the waketype shear flow leads to periodic vortex shedding, it can also be considered as a form of nonlinear waves. Thus, the present work also relates to the vortex dislocation problem in nonlinear waves.

The outline of the article is as follows. In Sec. II, as the beginning part, the wake-type upstream profiles with different spanwise disturbances are introduced, then mathematical formulation and numerical method for DNS are described. In Sec. III, numerical results and analysis of three distinct types of vortex dislocation are presented. In Sec. IV, some conclusions and discussions are made.

\section{NUMERICAL SIMULATIONS}

\section{A. Wake-type upstream velocity profiles}

In the present DNS computations a basic twodimensional wake velocity profile with spanwise threedimensional disturbance superimposed is taken as the streamwise upstream flow. The two-dimensional wake velocity profile is taken from the time-averaged streamwise velocity profile at the position in the near wake close to the cyl- inder, obtained by referring to previous DNS results ${ }^{13,14}$ and the experimental measurements ${ }^{15}$ of a cylinder wake flow. As discussed before, the profile flow has been shown to be absolutely unstable, resulting in a Kármán vortex street.

In the Cartesian system, $x, y$, and $z$ are coordinates defined in the streamwise, vertical, and spanwise directions, respectively. The general dimensionless expression of the wake-type upstream velocity profiles with spanwise disturbances can be written as

$$
U(y, z)=1.0-a(z)\left\{2.0-\cosh \left[(b y)^{2}\right]\right\} \exp \left[-(c y)^{2}\right],
$$

where $a(z), b$, and $c$ are parameters determining various shapes of wake-type upstream velocity profiles with different types of spanwise disturbances, as shown in the following context. The term $a(z)$ is the velocity defect factor. The variables of length and velocity have been normalized, respectively, by the characteristic length $D$ and velocity $U_{\infty} . D$ is determined by $U(D / 2)=\frac{1}{2}[U(0, \infty)+U(\infty, \infty)]$, while $U_{\infty}$ is the upstream flow velocity at $y= \pm \infty$.

In the present study of wake-type flow evolution three typical types of spanwise disturbance on the velocity profile are introduced. In type I, a local exponential decay disturbance is imposed at the center of the span which decays exponentially with the increase in spanwise distance. The parameters are

$$
a(z)=1.1+0.4 \exp \left(-z^{2}\right), \quad b=1.1, \quad c=1.2 .
$$

In type II, a stepped spanwise variation of velocity defect is introduced at spanwise positions. That is

$$
\begin{aligned}
& a(z)=1.1, \quad b=1.1, \quad c=1.2 \quad \text { if }|z|>2.5, \\
& a(z)=1.1, \quad b=0.9, \quad c=0.918 \quad \text { if }|z| \leq 2.5 .
\end{aligned}
$$

In type III, a spanwise sinusoidal wavy disturbance with various amplitudes across the span is imposed on the velocity defect of the upstream flow,

$$
a(z)=1.1+A \cos (2 \pi n z / L), \quad b=1.1, \quad c=1.2,
$$

where $L$ is the nondimensional spanwise size of computational domain, $A$ is the nondimensional wave height of disturbance, and $n$ is the number of disturbance wave. Then the wavelength of disturbance is obtained as $\lambda=L / n$. Correspondingly, the wave steepness is defined by $A / \lambda$.

\section{B. Theoretical formulation and numerical method}

In order to obtain high numerical accuracy and wavenumber resolution a compact finite difference-Fourier spectral hybrid method is used for solving three-dimensional Navier-Stokes (NS) equations. A detailed presentation of these equations and the numerical method can be found in Refs. 16 and 17. The procedure of the method is summarized briefly as follows. In the spanwise direction of the flow, the periodic boundary conditions are assumed, and then all the flow variables $(\varphi)$ are expanded into a truncated Fourier series as 


$$
\varphi(x, y, z, t)=\sum_{m=-N / 2}^{N / 2-1} \varphi_{m}(x, y, t) e^{-i m \beta z},
$$

where $N$ is the cutoff and $\beta$ is the spanwise wavenumber. The three-dimensional incompressible NS equations are written in dimensionless variable formulation as

$$
\frac{\partial \boldsymbol{V}}{\partial t}+(\boldsymbol{V} \cdot \nabla) \boldsymbol{V}=-\nabla p+\frac{1}{\mathrm{Re}} \nabla^{2} \boldsymbol{V},
$$

where $\boldsymbol{V}$ is the velocity vector, $(u, v, w)$ are the velocity components in streamwise, vertical, and spanwise directions, respectively, and $p$ is the pressure. The real time $t^{*}$ and pressure $p^{*}$ are normalized by $D / U_{\infty}$ and dynamic head, respectively. The Reynolds number is defined as Re $=U_{\infty} D / \nu$, where $\nu$ is the kinematic viscosity. Substituting Eq. (5) into Eq. (6), a system of equations for the mth harmonic in a two-dimensional $(x, y)$ domain can be obtained,

$$
\frac{\partial \boldsymbol{V}_{m}}{\partial t}+F_{m}[(\boldsymbol{V} \cdot \nabla) \boldsymbol{V}]=-\nabla_{m} p_{m}+\frac{1}{\operatorname{Re}} \nabla_{m}^{2} \boldsymbol{V}_{m},
$$

where $\nabla_{m}=\partial / \partial x, \partial / \partial y,-i m \beta, \quad \nabla_{m}^{2}=\partial^{2} / \partial x^{2}+\partial^{2} / \partial y^{2}-m^{2} \beta^{2}$, and $F_{m}[(\boldsymbol{V} \cdot \nabla) \boldsymbol{V}]$ is the Fourier transformation of the nonlinear terms. For the time discretization of the equation, following the work of Karniadakis et al., ${ }^{18}$ a third-order mixed explicit-implicit schemes is used. The solution procedure at every time step can be split into the following three substeps:

$$
\begin{aligned}
& \frac{\boldsymbol{V}_{m}^{\prime}-\sum_{q=0}^{J_{i}-1} \alpha_{q} \boldsymbol{V}_{m}^{n-q}}{\Delta t}=-\sum_{q=0}^{J_{e}-1} \beta_{q} F_{m}\left[(\boldsymbol{V} \cdot \nabla) \boldsymbol{V}^{n-q}\right], \\
& \frac{\boldsymbol{V}_{m}^{\prime \prime}-\boldsymbol{V}_{m}^{\prime}}{\Delta t}=-\nabla_{m} p_{m}^{n+1}, \\
& \frac{\gamma_{0} \boldsymbol{V}_{m}^{n+1}-\boldsymbol{V}_{m}^{\prime \prime}}{\Delta t}=\frac{1}{\operatorname{Re}} \nabla_{m}^{2} \boldsymbol{V}_{m}^{n+1},
\end{aligned}
$$

where $\boldsymbol{V}_{m}^{\prime}$ and $\boldsymbol{V}_{m}^{\prime \prime}$ are intermediate values of velocity, $J_{i}$ and $J_{e}$ are parameters for the order of scheme, and $\alpha_{q}, \beta_{q}$, and $\gamma_{0}$ are appropriately chosen weights. To evaluate the nonlinear terms in the split equations, the pseudospectral method is adopted, and the fifth order upwind compact scheme is used to approximate the terms. For solving the Helmholtz equations for pressure and velocity, a nine-point compact scheme of fourth-order accuracy is derived as follows (Refs. 16 and 17):

$$
\begin{aligned}
& \frac{10\left(\varphi_{2}+\varphi_{4}\right)-2\left(\varphi_{1}+\varphi_{3}\right)+\left(\varphi_{5}+\varphi_{6}+\varphi_{7}+\varphi_{8}\right)-20 \varphi}{(\Delta y)^{2}} \\
& -\frac{10\left(\varphi_{1}+\varphi_{3}\right)-2\left(\varphi_{2}+\varphi_{4}\right)+\left(\varphi_{5}+\varphi_{6}+\varphi_{7}+\varphi_{8}\right)-20 \varphi}{(\Delta x)^{2}} \\
& +\left(8 \varphi_{0}+\varphi_{1}+\varphi_{2}+\varphi_{3}+\varphi_{4}\right) b=\left(8 f_{0}+f_{1}+f_{2}+f_{3}+f_{4}\right),
\end{aligned}
$$

and sixth-order center compact scheme is used for nonhomogeneous term $(f)$ in calculations.

\section{Boundary conditions}

For the numerical simulation of the open space flows, correct treatment of the boundary conditions is very important because it not only directly affects the overall accuracy of the scheme but also determines the efficiency of the timestepping algorithm. At the entry of the computational domain, the boundary conditions are given by the imposed velocity profile (1). On the upper and lower free boundaries the boundary conditions for velocity are

$$
\frac{\partial u}{\partial y}=0, \quad v=0, \quad w=0 .
$$

For outlet boundary conditions in far downstream, it may be assumed that the flow has a preferred direction along the $x$ axis and goes through freely the downstream boundary without any nonphysical feedback. However these type conditions will lead to a large amount CPU time and memory storage. If the size of computation domain is not large enough, a nonreflecting-type outlet boundary condition is needed which is required to deal with both the nonlinear convection and viscous diffusive mechanism of the flow that well matches the NS equations adopted inside the domain. This approach minimizes the spurious perturbation waves propagated upstream. For these reasons a form of nonreflecting outlet conditions were used which is an analog of the classical wave equation, suggested by Jin and Braza ${ }^{19}$ and generalized to three-dimensional case by our previous studies $^{16,17}$ (it is noticed that the similar result was also obtained by Persillon and $\mathrm{Braza}^{20}$ ). The nonreflecting boundary conditions at the outlet position of the computation domain are formulated as

$$
\frac{\partial \boldsymbol{V}}{\partial t}+u \frac{\partial \boldsymbol{V}}{\partial x}=\frac{1}{\operatorname{Re}}\left(\frac{\partial^{2} \boldsymbol{V}}{\partial y^{2}}+\frac{\partial^{2} \boldsymbol{V}}{\partial z^{2}}\right) .
$$

The corresponding spectral form used in the computation is

$$
\frac{\partial \boldsymbol{V}_{m}}{\partial t}+F_{m}\left[u \frac{\partial \boldsymbol{V}}{\partial x}\right]=\frac{1}{\operatorname{Re}}\left(\frac{\partial^{2} \boldsymbol{V}_{m}}{\partial y^{2}}-m^{2} \beta^{2} \boldsymbol{V}_{m}\right) .
$$

Equation (12) must be solved simultaneously with the thirdorder mixed explicit-implicit schemes adopted for NS equations in the inner domain.

For the pressure boundary conditions from Eqs. (7) and (8), a semidiscretized pressure boundary condition can be expressed as

$$
\begin{aligned}
\frac{\partial p_{m}^{n+1}}{\partial n}= & \boldsymbol{n} \cdot\left\{-\frac{\partial \boldsymbol{V}_{m}}{\partial t}+\frac{1}{\operatorname{Re}} \nabla_{m}^{2} \boldsymbol{V}_{m}^{n+1}\right. \\
& \left.-\sum_{q=0}^{J_{e}-1} \beta_{q} F_{m}\left[\left(\boldsymbol{V}^{n-q} \cdot \nabla\right) \boldsymbol{V}^{n-q}\right]\right\} \\
= & \boldsymbol{n} \cdot\left\{-\frac{\partial \boldsymbol{V}_{m}}{\partial t}-\frac{1}{\operatorname{Re}} F_{m}\left[\nabla \times \boldsymbol{\Omega}^{n}\right]\right. \\
& \left.-\sum_{q=0}^{J_{e}-1} \beta_{q} F_{m}\left[\left(\boldsymbol{V}^{n-q} \cdot \nabla\right) \boldsymbol{V}^{n-q}\right]\right\},
\end{aligned}
$$

where $\nabla^{2} \boldsymbol{V}=\nabla D-\nabla \times \boldsymbol{\Omega}, \boldsymbol{\Omega}=\nabla \times \boldsymbol{V}$, and $D=\nabla \cdot \boldsymbol{V}$ are used. 
By forcing $D^{n+1} \equiv 0$, in each time step and replacing $\nabla$ $\times \boldsymbol{\Omega}^{n+1}$ by $\nabla \times \boldsymbol{\Omega}^{n}$, the divergence-free constraint can be well satisfied on the boundaries.

In computation, the parameters are as follows. Reynolds number is taken as 200. The nondimensional sizes of the computational domain are 100, 30, and 30 in streamwise, vertical, and spanwise directions, respectively, used for type I. For saving computer resources in types II and III, the computational domain becomes 60,30 , and 30 , respectively. The cutoff of the truncated Fourier series is $N=64$. The corresponding grid points in the $x-y$ plane are $202 \times 62$ in the case of the type I, and $122 \times 62$ in the cases of types II and III. The reliability of the numerical code used in the present work is verified first through a preliminary calculation of a two-dimensional time-average flow. The velocity profile used in computation is obtained from the DNS results of two-dimensional wake flow with cylinder at $\operatorname{Re}_{d}=100\left(\operatorname{Re}_{d}\right.$ is defined by $U_{\infty} d / \nu, d$ is the cylinder diameter). ${ }^{14}$ The corresponding parameters in velocity profile, Eq. (1), are $a=1.3$, $b=0.5$, and $c=0.75$, respectively. The computational results reproduce a normal two-dimensional Kármán vortex street. The computed Strouhal number is 0.189 , whereas behind the cylinder it was $0.179 .{ }^{14}$ The present result compares well with $\mathrm{St}=0.195$ calculated by a time-average profile flow obtained by Triantafyllou and Karniadakis ${ }^{13}$ at same Reynolds number. The above quantitative agreement is satisfactory. The small shift in calculated frequency may be attributed to the effect of the inflow upstream boundary condition. The preliminary computations also show that as the computational domain (say in $x$ direction) changes from 60 to 100 and the Fourier mode varies from 64 to 128, the flow structures calculated are almost unchanged. The difference in fluctuation velocity between the two cases is very small, and the absolute error for these two cases is of $O\left(10^{-6}\right)$.

\section{NUMERICAL RESULTS}

DNS of the evolution of wake-type upstream flows with three different spanwise disturbances has been carried out. Three distinct modes of vortex dislocation and wake behavior have been found in the wake flows. In Secs. III A-III C, the general characteristics of spatial-temporal evolution of different upstream flows and three distinct modes of vortex dislocation produced in wakes will be described, respectively. From the point of view of substantial modification of vorticity, by examining the variations of vortex lines and vorticity distribution, the physical processes of the vortex dislocation development and the complex vortex linking over the dislocation are illustrated. Different mechanisms responsible for the presence of vortex dislocations are discussed. The effect of vortex dislocation on wake transition and the unsteadiness of wake behavior are reported, respectively. It should be mentioned that the present results of wake flow structure differ from those induced by the natural threedimensional instability of flow around a circular cylinder, that is mode A three-dimensional vortex structure that occurs at the Reynolds number of 180 and above. ${ }^{2}$ In the present study at the Reynolds number of computations the wake remains two dimensional in the absence of three-dimensional

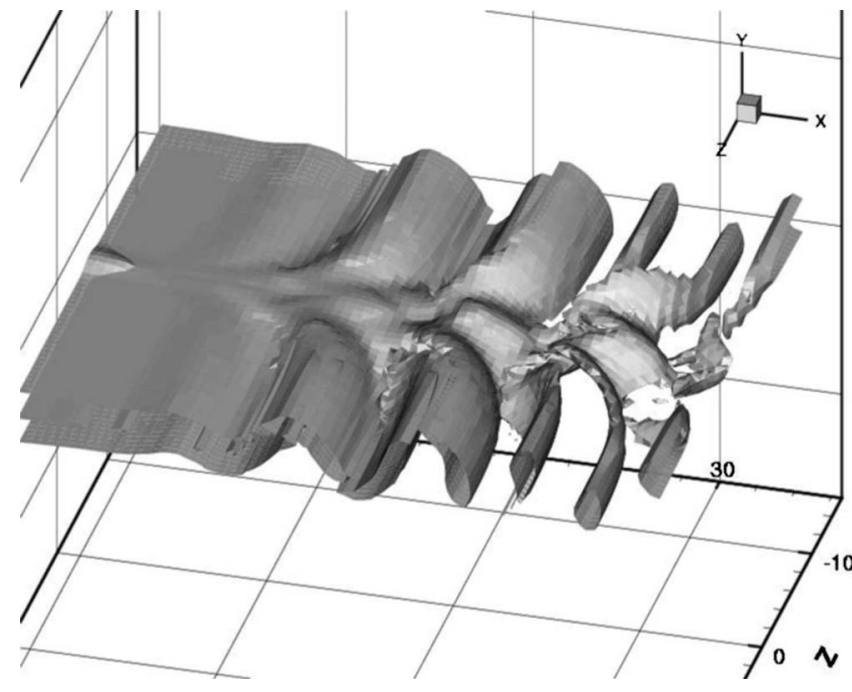

FIG. 1. Upstream isosurfaces of spanwise vorticity $\left|\omega_{z}\right|=0.15$ at $t=260$.

disturbances. Three-dimensional evolution and flow structures of the wake-type flows, being described below, are determined by the imposed three-dimensional disturbances. Mode A flow pattern was not observed although the computation Reynolds number is nearly the same.

\section{A. Local spanwise exponential decay disturbance}

For this case the flow instability development and vortex rolling up are nonsynchronous along the span. In the early stage of flow evolution an oblique mode of vortex shedding occurs across the span, as shown in Fig. 1. As time increases, a systematic twisted chainlike mode of vortex dislocation is generated in the middle downstream caused by the local spanwise disturbance in the upstream flow. The overall vortex flow pattern, identified by $\lambda_{2}$-definition, ${ }^{21}$ is shown in Fig. 2. The vortex dislocation is symmetric about the central plane $(z=0)$ and occurs consecutively on the spanwise vortex tubes of base flow. It is characterized by spanwise vortex branching and complex vortex linking between vortices. The numerical visualization in Fig. 2 suggests a typical mode of vortex dislocation whereby at a certain spanwise position, a spanwise vortex is divided and linked, back and forth, into two vortices with opposite signs on the same side. Significant undulation of the spanwise vortex tubes is clearly seen in the region near the center of the span. Near the center the

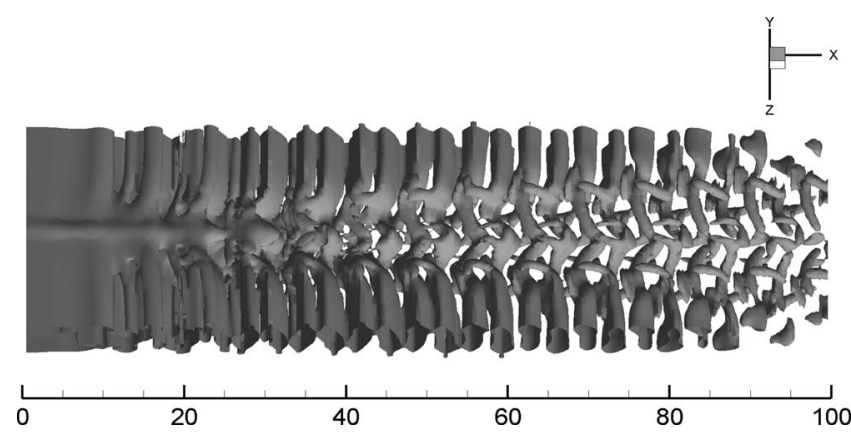

FIG. 2. The overall flow pattern with symmetric twisted chainlike vortex dislocations identified by $\lambda_{2}$-definition $\left(\lambda_{2}=-0.003\right), t=260$. 


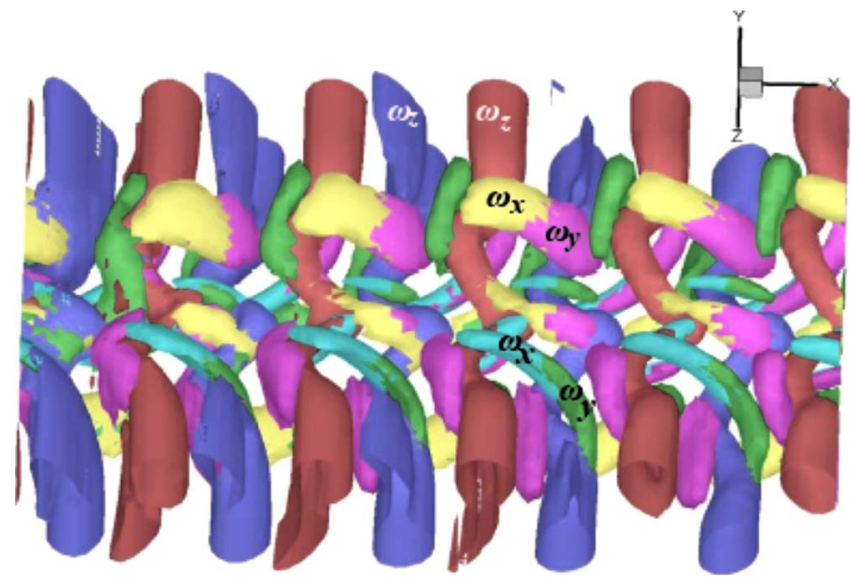

FIG. 3. (Color online) Topology of vortex linking between three components of vorticity in vortex dislocation, $t=260$. Iso-surfaces of vorticity: $\left|\omega_{x}\right|=0.1,\left|\omega_{y}\right|=0.06,\left|\omega_{z}\right|=0.12$.

undulated spanwise vortex is attached by two streamwise vortex branches diverted from the spanwise vorticity. Spatial distribution of three components of vorticity and their connections in vortex dislocation are shown in Figs. 3 and 4, respectively. These calculated results clearly show further the fundamental modification of vorticity from spanwise direction to streamwise and vertical directions and the vortex linking between different vortices in the vortex dislocations. Some samples of calculated vorticity distribution in the wake are shown in Figs. 5 and 6. In the figures, the spanwise spreading and vertical development of the vortex dislocation can also be recognized clearly.

In order to understand the physical processes of the development of vortex dislocation and identification of vortex

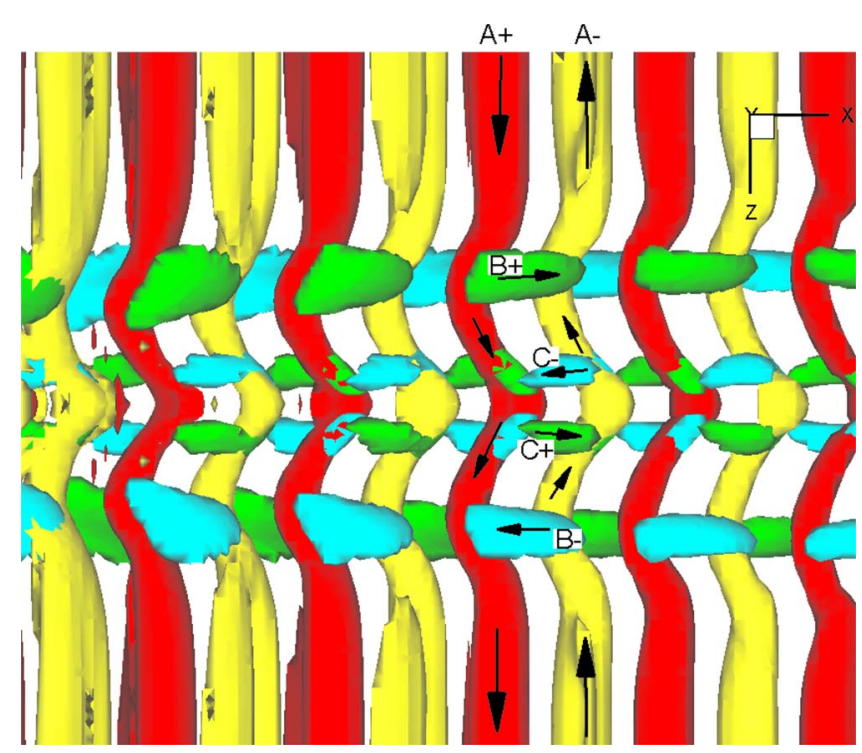

FIG. 4. (Color online) Top view of vortex linking between spanwise and streamwise vorticity in the vortex dislocations at certain middle downstream positions. $A_{+}$and $A_{-}$represent spanwise vortices with positive and negative signs, respectively. $B$ and $C$ show the streamwise vorticity branches diverted from the spanwise vortices at two positions with the largest phase differences in spanwise vortices. The vortex linking, back and forth, of two components of vorticity is shown in the figure at $t=260$.

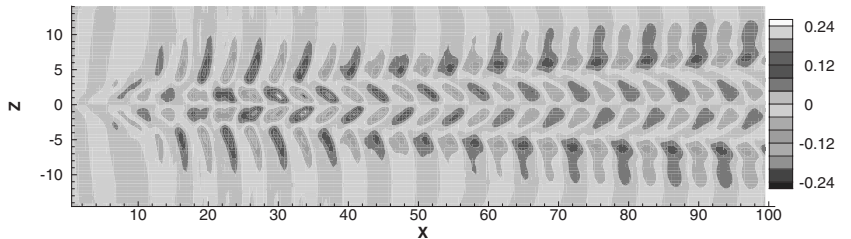

(a)

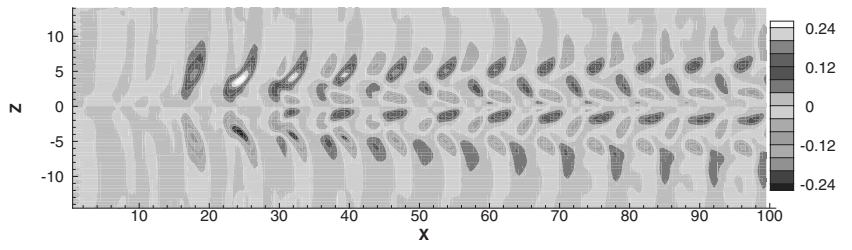

(b)

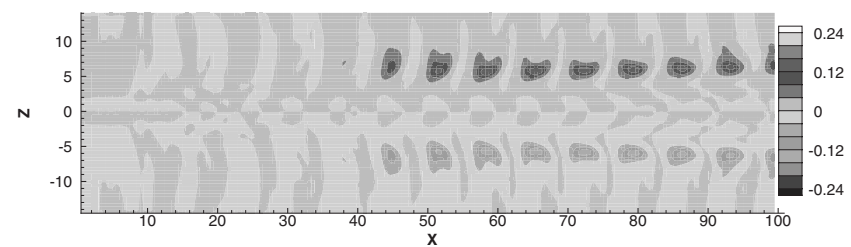

(c)

FIG. 5. Distributions of streamwise vorticity $\omega_{x}$ at different vertical planes, $t=260$, (a) $y=0$; (b) $y=-1.5$; (c) $y=-4$.

linkages over the dislocation, the variation of several representative vortex lines emitted from a primary spanwise vortex is examined in detail. As shown in Figs. 7-9, from upstream to downstream, the vortex line tracks are changed successively from nearly parallel, to spanwise direction with a small undulation in the center region, to noticeable

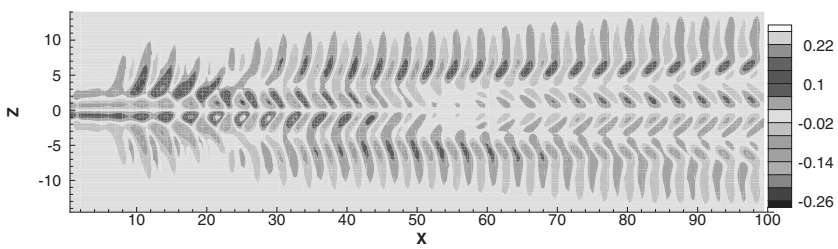

(a)

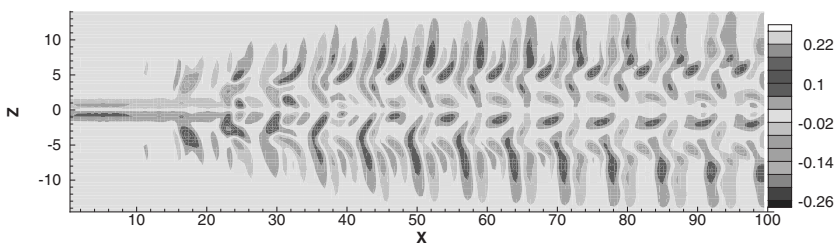

(b)

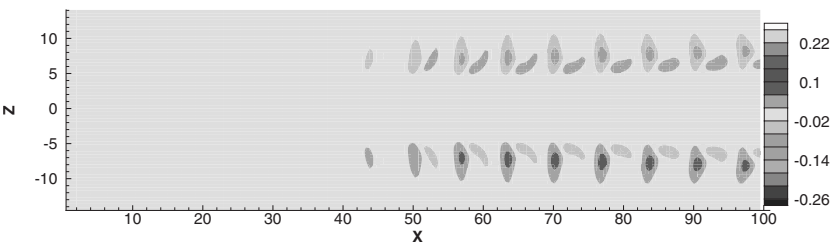

(c)

FIG. 6. Distributions of vertical vorticity $\omega_{y}$ at different vertical planes, $t$ $=260$, (a) $y=0$; (b) $y=-1.5$; (c) $y=-4$. 


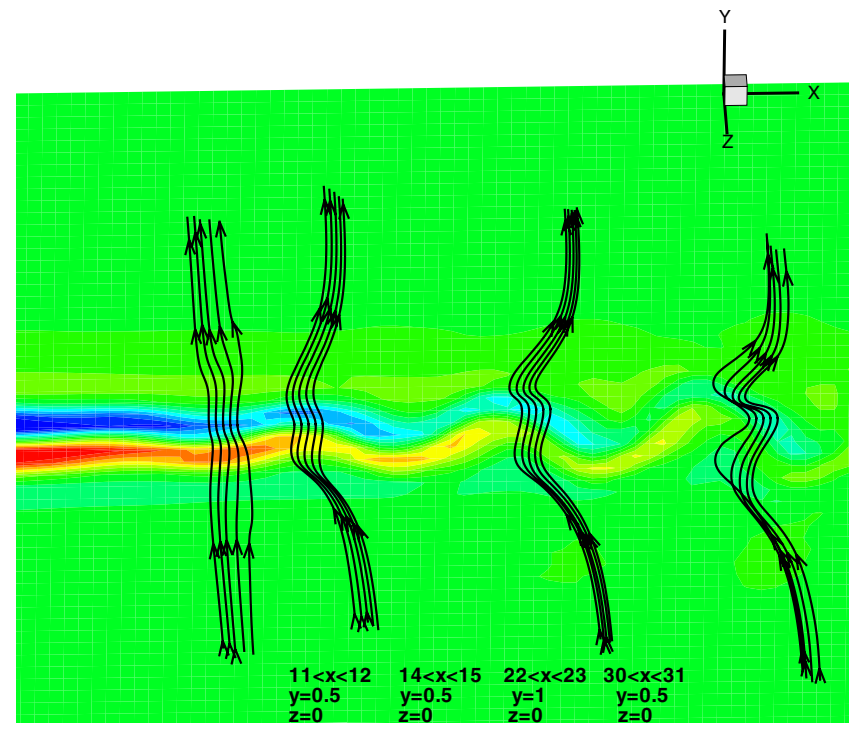

FIG. 7. (Color online) Variations of vorticity lines at different downstream positions. The lines pass through several places denoted by the coordinates $(x, y, z)$ in the diagram. The background is a spanwise vorticity pattern at the plane of $z=-15$.

spanwise undulation. As the flow travels downstream, this undulated distortion of vortex lines becomes much stronger. In the middle downstream, some vortex lines, usually in the outer region of a spanwise vortex tube, are separated from the primary spanwise vortex and then turned to the adjacent spanwise vortices with opposite signs, becoming a part of them on the same side. Further downstream, in addition to the spanwise vortex splitting and distortion, some split vortex lines are wound back and forth around other vortices. A detailed vortex linkage between adjacent spanwise vortices over the dislocation is illustrated in Fig. 9 by the vortex line tracks. It shows that in the dislocation the vortex elements

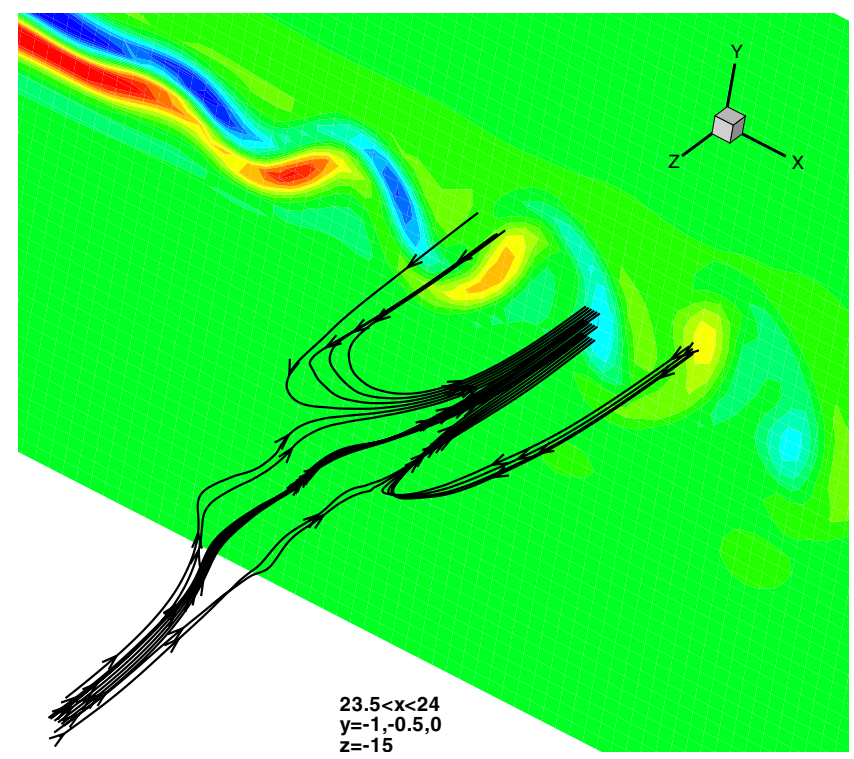

FIG. 8. (Color online) Spanwise vortex twisting, vortex splitting, and reconnection represented by the variations of vorticity lines passing through the locations denoted by the coordinates $(x, y, z)$ in the diagram. The background is a spanwise vorticity pattern at the plane of $z=-15$.

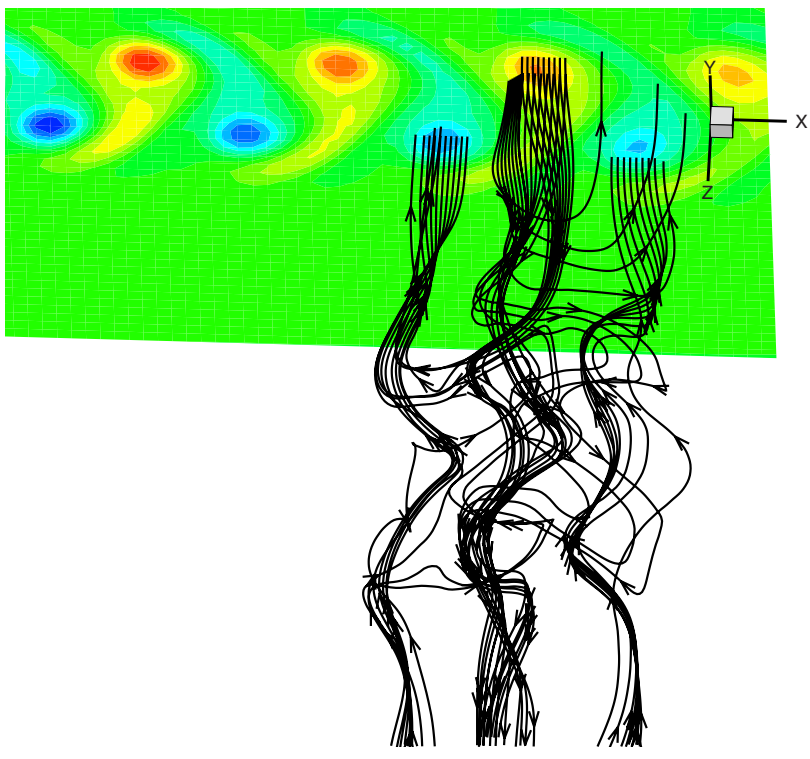

(a)

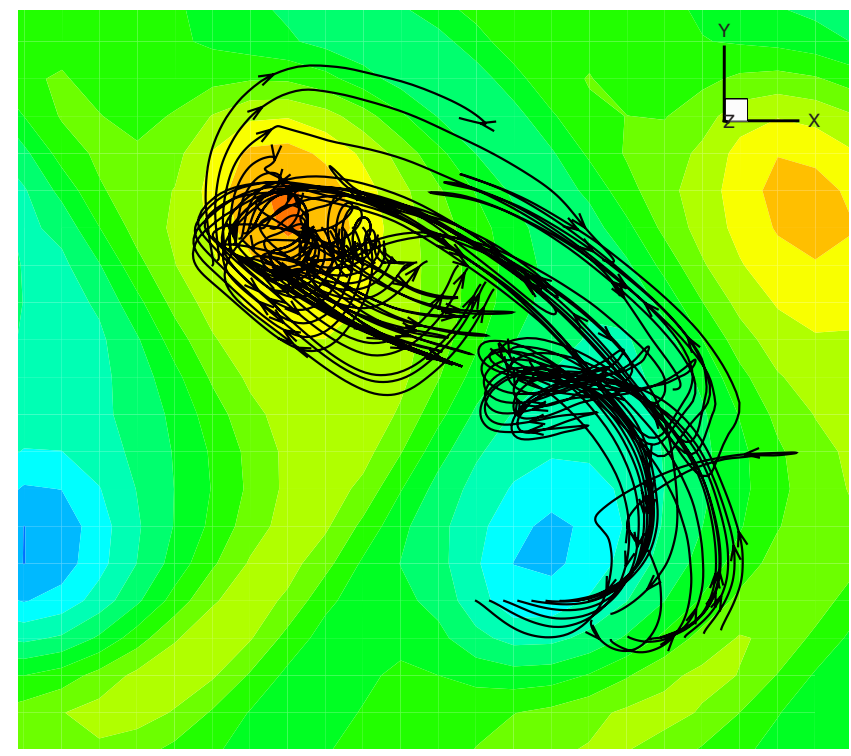

(b)

FIG. 9. (Color online) Behavior of vortex lines among three adjacent spanwise vortices in the chainlike vortex dislocations at $t=260$. In the figure vortex lines emitted from one side of a spanwise vortex (in the middle) and two adjacent vortices are drawn. Spanwise vortex twisting, vortex branching, complex linking, as well as vortex line winding around the other vortices are illustrated. (a) Three-dimensional view; (b) the end view.

are highly distorted in three dimensions, and the vortex line tracks present a disorderly aspect. From the successive stages of the variation of vortex lines, it is known that the whole physical process of development of vortex dislocation includes the oblique shedding of spanwise vortices upstream, with the shed vortex twisting and distortion a little downstream and then the spanwise vortex splitting and reconnection further downstream, resulting in a highly threedimensional vortex dislocation structure. The nature of the vortex dislocation is the substantial modification of vorticity from original spanwise to streamwise and vertical directions. 


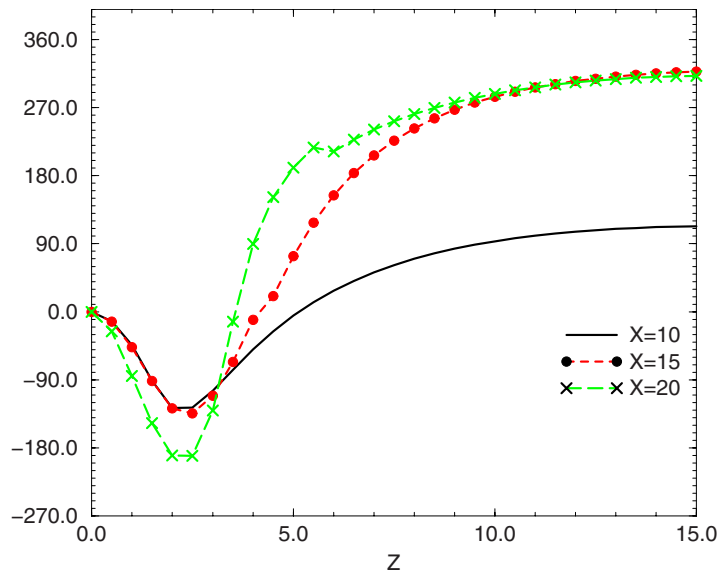

(a)

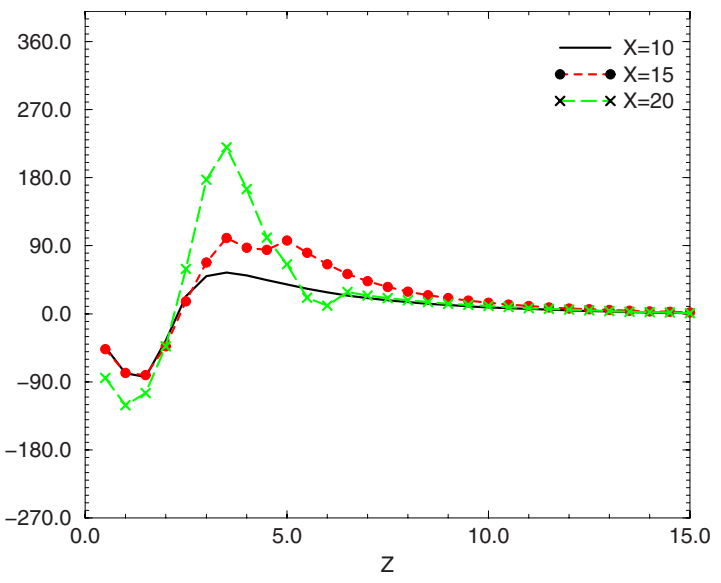

(b)

FIG. 10. (Color online) (a) The phase variation of spanwise vortex with spanwise direction at different streamwise positions. (b) The phase difference in spanwise vortex along the spanwise direction.

The present calculation also shows that the local spanwise exponential decay disturbance in upstream flow causes a spanwise variation of vortex strength. Actually the initial vortex strength can be evaluated approximately by the formula $\Gamma(z) \sim\left[\int_{-\infty}^{+\infty} d y \int_{0}^{l} \omega_{z} d x\right] \cdot \eta$, where $l$ and $\eta$ are assumed as a unit length in streamwise direction and the vorticity dissipation coefficient, respectively. Result shows that $\Gamma(z)$ is proportional to the spanwise velocity defect factor $a(z)$. It decreases exponentially from the highest value at the center of the span to a low constant value at the positions away from the center. The variation of the strength in the spanwise vortex tube will result in spanwise spreading of the vortex dislocation structure and further twisted deformation of vortex tubes in dislocation. These results are consistent with a related analysis suggested previously by Leonard. ${ }^{22}$ Of particular interest, the local spanwise exponential disturbance causes a remarkable phase difference between the spanwise vortex shedding cells, as shown in Fig. 10. Particularly at the two spanwise positions of $|z|=1.5$ and 3.5 , the rate of phase variation reaches two different extreme values. By examining vortex patterns (Fig. 2) and the vorticity distributions of the streamwise and vertical components (Figs. 4-6), it is known that just at these two locations the spanwise vortex splitting and reconnection occur producing streamwise and vertical components of vorticity. Therefore, it is clear that in the present case of upstream flow the vortex dislocation is mainly caused by the significant phase difference between shedding cells.

The effect of vortex dislocation on velocities in the wake is studied in detail. Both the fluctuating and mean velocities are significantly influenced by the appearance of the chainlike vortex dislocation. The features of velocity contours are closely associated with the formation of the dislocation and the vortex linkages over the dislocation. They behave no longer as a pure time-averaged profile of streamwise velocity appearing in the normal wake. Contour of vertical fluctuating velocity in Fig. 11 has a large spanwise undulation across the center region, just like the aspect of the spanwise vortex tube. The vortex dislocation induces larger spanwise veloc- ity, resulting in spanwise expanding of the velocity contour (Fig. 12) downstream. The streamwise fluctuating velocity profile $u_{\text {rms }}$ in Fig. 13 exhibits two sets of different peak values, which are corresponding to the appearance of vortex dislocation structure in the center region and to a normal Kármán vortex shedding at both sides of the span, respectively. The larger fluctuation level in the center region profile is found at the middle downstream positions where the vortex tube likely starts dividing. As the flow travels downstream, the mean velocity profile at the center region of the span changes from a wake-type distribution upstream to almost jetlike shapes at certain downstream positions, as shown in Fig. 14. This is a feature shown in the behavior of this wake-type flow transition. Very similar behavior of the variation of mean velocity profile can be found in the cylinder wake transition with spotlike vortex dislocations shown by Williamson. ${ }^{1}$ Pronounced irregularities in time history of fluctuating velocity are displayed at certain downstream positions, which is caused by the appearance of vortex dislocations. Some examples are shown in Figs. 15(a) and 15(b). Correspondingly in the spectra, a fundamental frequency appeared at $f_{0}=0.1465$, which relates to spanwise vortex shedding, and is nearly the same across the span. Several pronounced harmonic peaks are found at $2 f_{0}$, and even at $4 f_{0}$ with a small energy. Again, the splitting of the spectra energy at lower and higher frequency values occurs in the vicinity of harmonics in the spectra of streamwise and vertical velocities. The phenomena of spectra energy split correspond to the

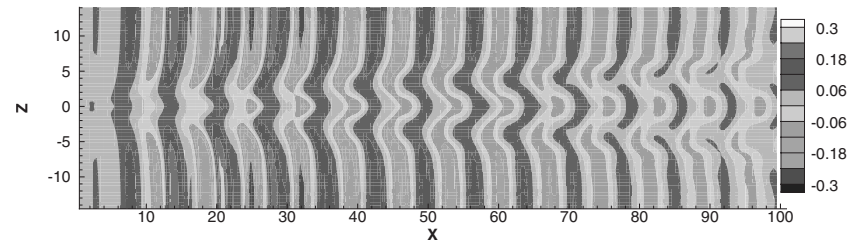

FIG. 11. Contours of vertical fluctuating velocity $v$ in the $x-z$ plane at $y$ $=0, t=260$. 


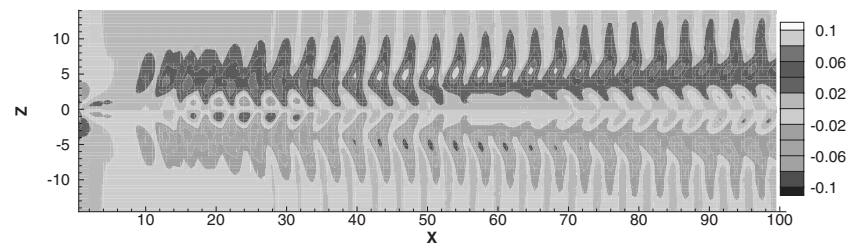

FIG. 12. Contours of spanwise fluctuating velocity $w$ in $x-z$ plane at $y=$ $-1, t=260$.

appearance of the vortex dislocation. Similar behavior has been reported in DNS investigations of natural vortex dislocation in the cylinder wake transition. ${ }^{10}$

The physical features described above, including the appearance of large-scale distorted vortex structures, highly three-dimensional distorted and disorderly tracks of vortex lines, as well as the pronounced irregularities in fluctuating velocity, characterize the flow transition from a regular laminar flow upstream to an unsteady irregular vortex flow with turbulent behaviors downstream.

\section{B. Stepped spanwise disturbance}

In the present case a large-scale spotlike mode of vortex dislocation is produced periodically in the center region of the span caused by the stepped spanwise disturbance on upstream flow. It can be seen from Fig. 16(a) that the vortex pattern is symmetric on either side of the spanwise center. The splitting of the original spanwise vortex appears at the position $(|z|=2.5)$ with the velocity step and the split vortex turning its direction to streamwise and then to adjacent spanwise vortices with opposite signs. Thus vorticity modification is from an original spanwise direction to streamwise and vertical directions, and then further to adjacent spanwise vortex with an opposite sign on the same side. The vortex reconnection takes place by a crossing vortex street mode. A typical mode of vortex linking in the dislocation is described by the variation of vortex line tracks, as shown in Fig. 17. These characteristics of vortex splitting and reconnection are the same as those appearing in chainlike vortex dislocations discussed in Sec. III A. However, in the present case the spotlike vortex dislocation is produced periodically in the streamwise direction, which is constituted within a time interval by several consecutive vortex splittings and reconnections. The periodicity of the appearance of the dislocation structure can also be identified by the distribution of streamwise vorticity downstream, as shown in Fig. 16(b). The vorticity modification and the periodicity of the occurrence of large-scale spotlike dislocations can be recognized from time sequences of flow pattern and the streamwise vorticity distributions.

The time series of velocity and the corresponding spectra at different spanwise positions are analyzed. Two samples of velocity $v$ component signal and its spectra at different spanwise positions are displayed in Figs. 18 and 19, respectively. It can be clearly seen that in the central region of $|z| \leq 3$, a pronounced modulation is found in the velocity signal (Fig. 18). The low frequency modulation corresponds to the passage of a vortex dislocation structure, while away from the

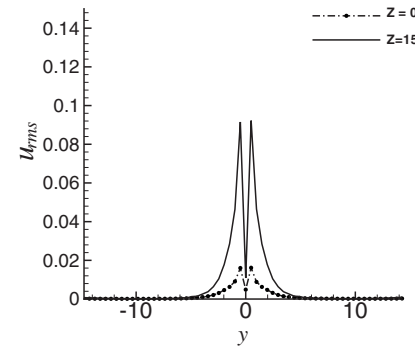

(a)

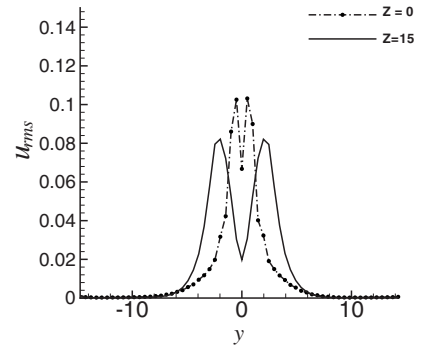

(b)

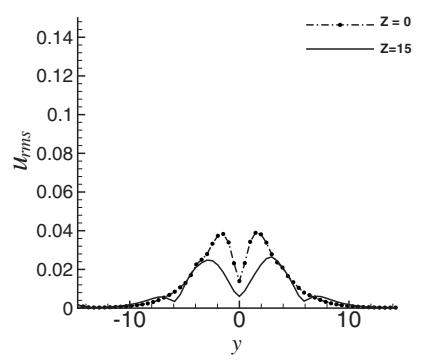

(c)

FIG. 13. Fluctuating velocity profiles $\left(u_{\mathrm{rms}}\right)$ (transverse) at different streamwise positions, $z=0$ and $z=15$, (a) $x=5$; (b) $x=25$; (c) $x=90$.

central region the signals exhibit a normal Kármán vortex shedding. Correspondingly, frequency analysis by fast Fourier transform shows two remarkable peak values at $f_{1}$ $=0.141$ and $f_{2}=0.117$ in the region of $|z| \leq 3$, while only one peak value $\left(f_{1}\right)$ is found for $|z|>4$. $f_{1}$ corresponds to the base flow fluctuation and $f_{2}$ is the shedding frequency in the nonuniform central region. Again, a peak value at $f_{b}=0.024$ is the beat frequency, equal to the difference in $f_{1}$ and $f_{2}$, caused by nonlinear interaction of the two frequencies. $f_{b}$ is the frequency of the occurrence of the spotlike vortex

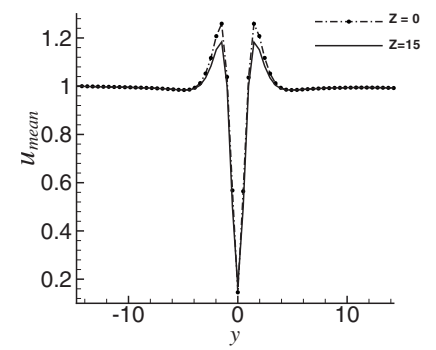

(a)

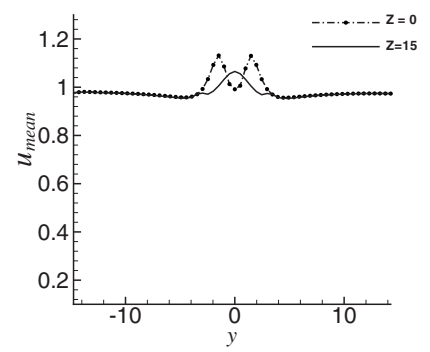

(b)

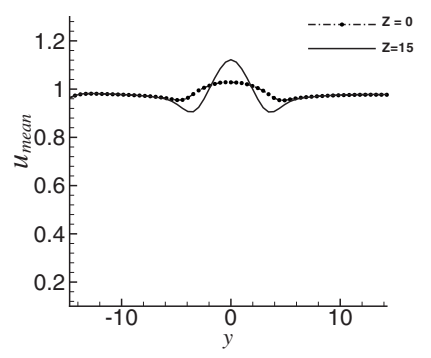

(c)

FIG. 14. Mean velocity profiles $\left(u_{\text {mean }}\right)$ (transverse) at different streamwise positions, $z=0$ and $z=15$, (a) $x=5$; (b) $x=25$; (c) $x=90$. 

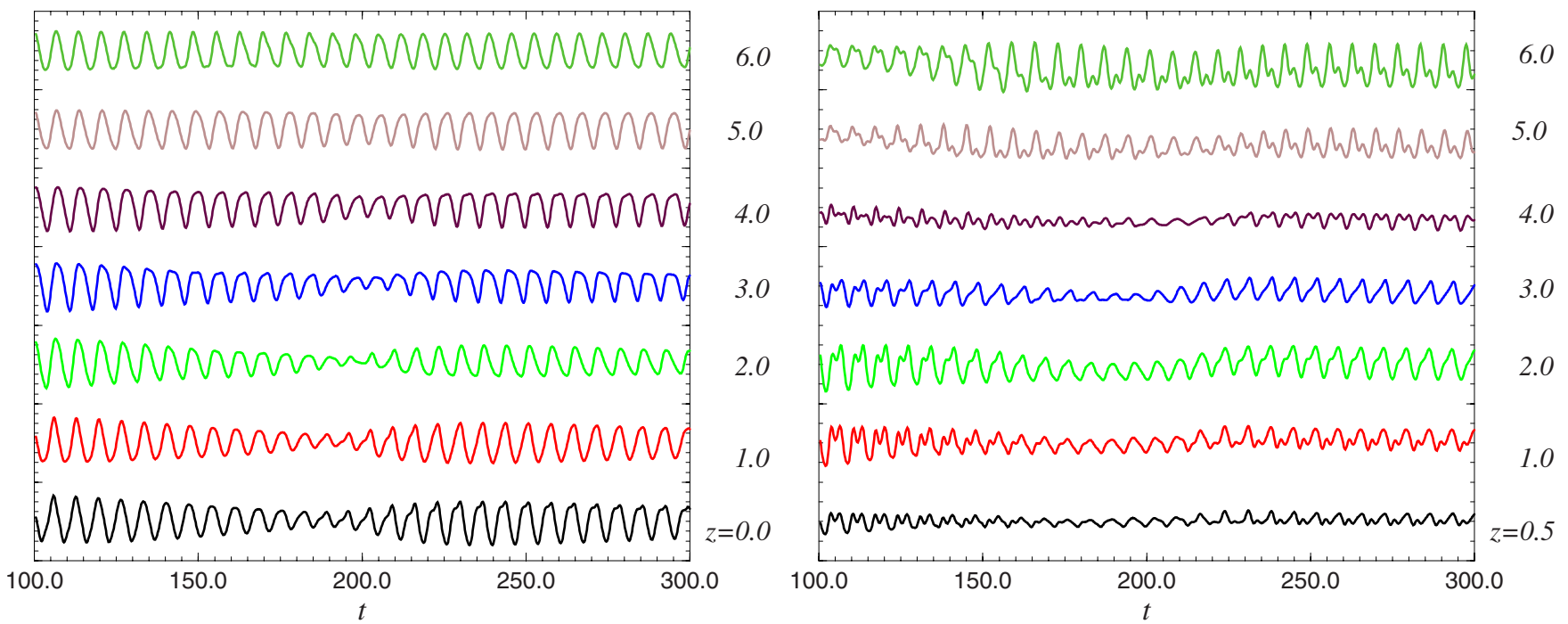

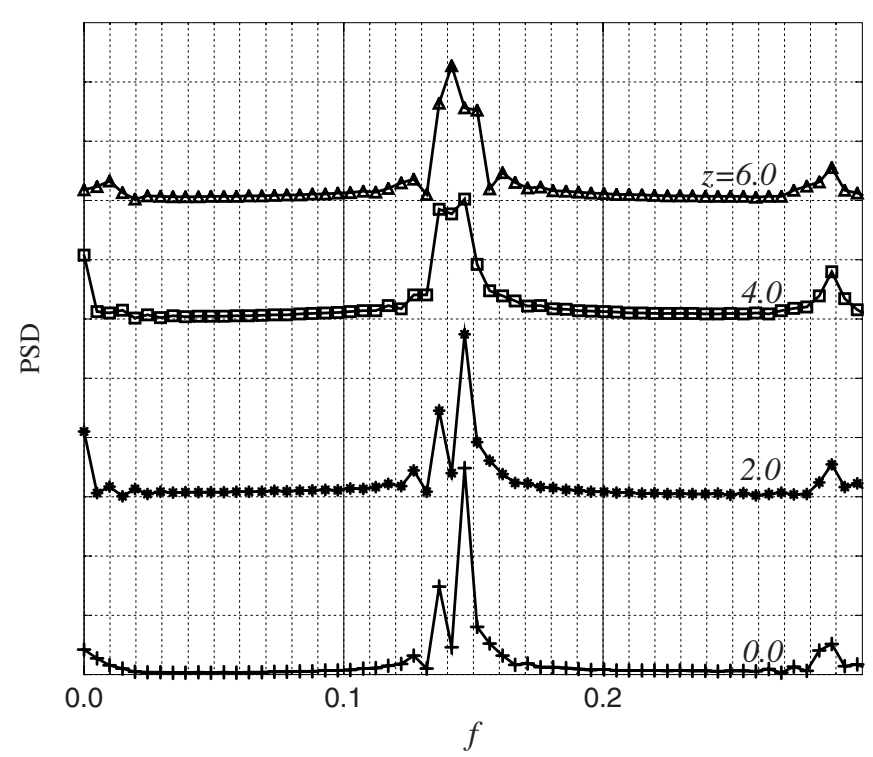

(a)

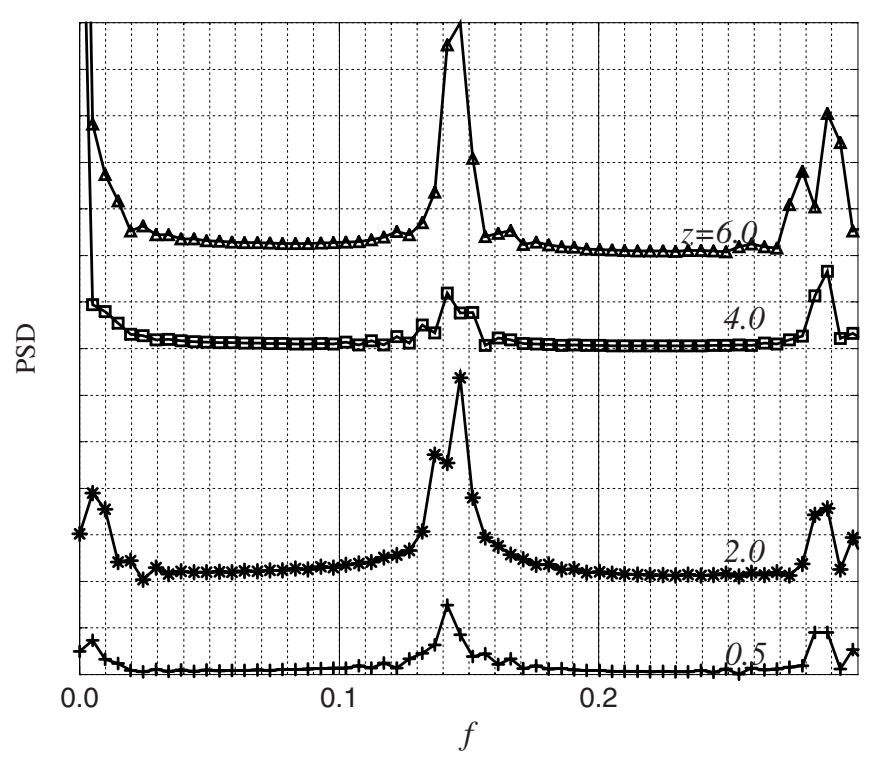

(b)

FIG. 15. (Color online) The time history and corresponding spectra of velocity components of (a) $v$ at position of $(x=70, y=1)$ and (b) $w$ at position of $(x$ $=80, y=1)$ at certain spanwise positions.

dislocation. Spectra of the spanwise velocity signal display very significant irregularities, as shown in Fig. 19. Many peak values at various frequencies are produced. By means of wavelet analysis of velocity variation it is found that the local frequency at certain downstream positions varies with time and spatial positions. The frequency broadening due to frequency interaction downstream is also pronounced. These physical features of large-scale vortex distortion and flow field irregularities characterize the behavior of the wake-type flow transition. In order to keep this paper short, the details of variation of frequency in wake are not discussed here. So far, from the above numerical results, it can be concluded that in the present case the spotlike vortex dislocation is caused by the frequency discontinuity appearing at the velocity steps on either side of the central region. The appearance of the vortex dislocation is a wake flow behavior that coordinates the frequency discontinuity in the span between the adjacent shedding cells, matching the vortex shedding flow on either side of the velocity steps. The basic features of the calculated vortex dislocation are very similar to those of indirect mode dominated flow pattern, produced at both sides of discontinuity in the diameter of a stepped cylinder, observed by Lewis and Gharib. ${ }^{3}$ Again, there are close similarities between the spotlike dislocations obtained by present numerical simulation and the symmetric two-side dislocations generated at either side of a small ring on a circular cylinder reported by Williamson's experiments. ${ }^{1}$ The similarities include the spanwise vortex dividing, the vorticity modification from spanwise to streamwise direction, and the complex reconnection between neighboring vortices with the opposite sign. It is demonstrated that there are some common features in the generation of the vortex dislocation and the vortex linkages over the dislocation between the wake-type flow and the cylinder wakes as the dislocation is produced by 

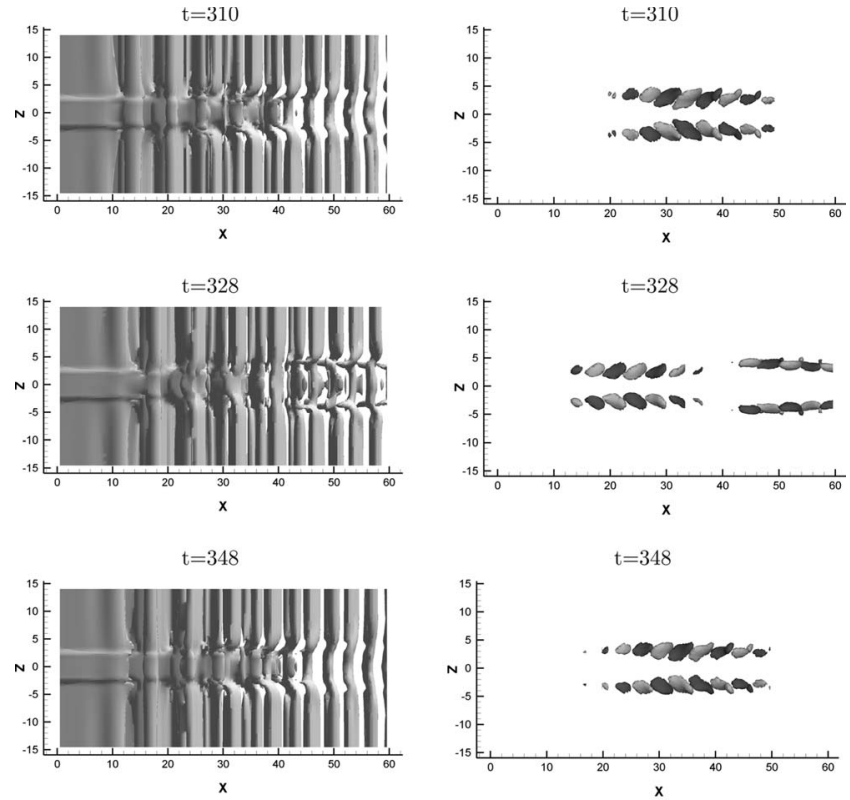

(a)

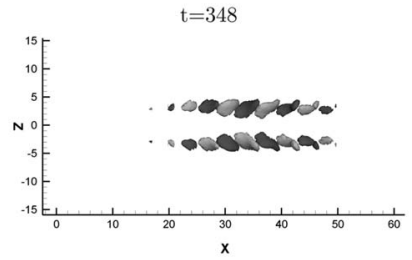

(b)

FIG. 16. Time sequence of large-scale spotlike modes of vortex dislocation, visualized by (a) $\lambda_{2}$-definition $\left(\lambda_{2}=-0.005\right)$ and (b) the distribution of streamwise vorticity, with the evolution of time at $t=310,328$, and 348, respectively. (a) shows that the spotlike vortex dislocation is produced periodically in the central region. In the vortex dislocation, spanwise vortex splitting and reconnection can be seen clearly. On the other hand, the streamwise vorticity component is mainly produced in the process of spanwise vortex splitting and reconnection, as shown in (b). These distributions also display the periodicity of the appearance of spotlike vortex dislocation pattern, as shown in (a).

the discontinuity of shedding frequencies, although there are different flow patterns caused by different flow conditions. The present numerical results provide detailed information of the vorticity modification in the wake-type flow with velocity steps.

The dynamics of spotlike modes of vortex dislocation in the wake-type flow was previously studied by Zhao and $\operatorname{Ling}^{23}$ by means of a low-dimensional dynamical system

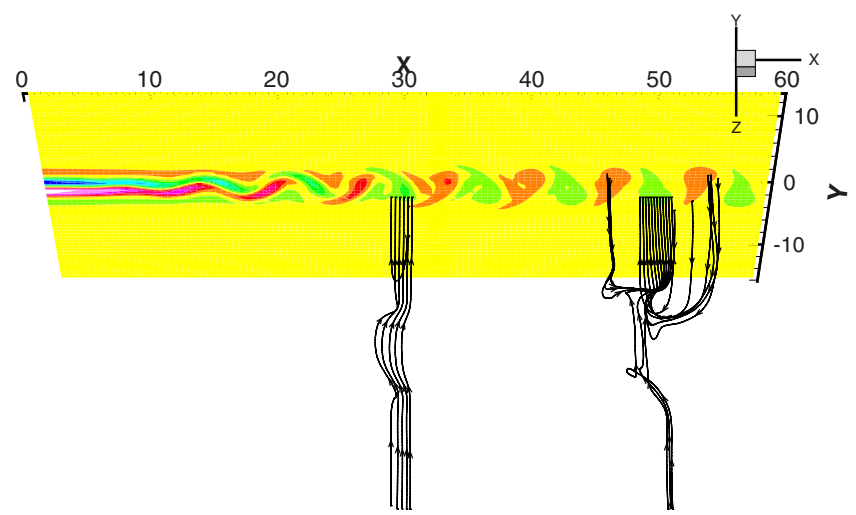

FIG. 17. (Color online) Vortex linking in the spotlike vortex dislocation structure illustrated by vortex line behavior at $t=328$. At the moment a spotlike dislocation structure is passing through at $x=52$, the spanwise vortex splitting and linking to its adjacent vortices occurred in the middle of the dislocation structure are shown. While at the position of $x=30$ vortex lines show the beginning aspect of another following spotlike dislocation.

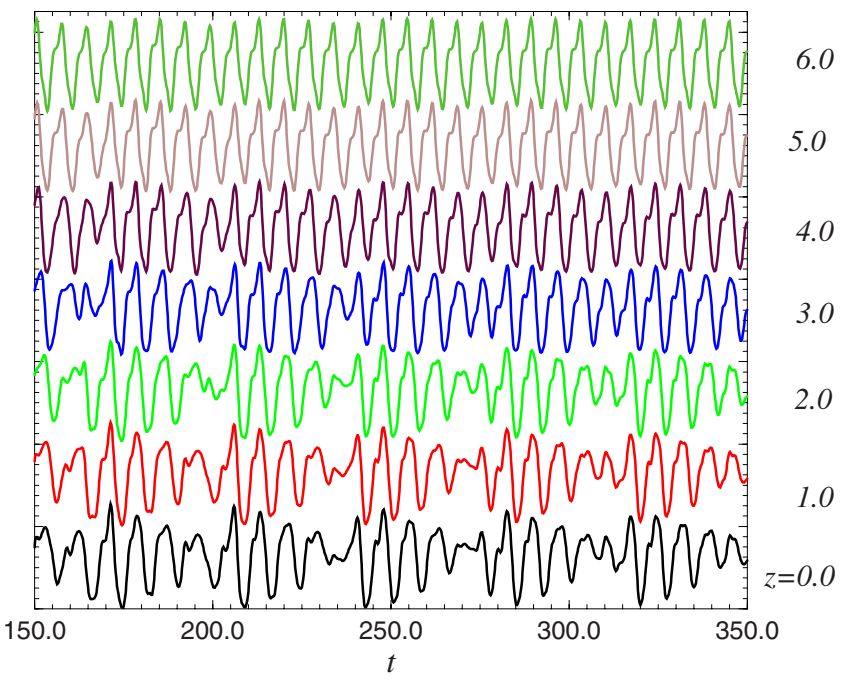

FIG. 18. (Color online) Time signals of vertical velocity component at different spanwise positions of $(x, y)=(55,0.5)$. In the central region of the span, $z \in(0,3)$, velocity signals show noticeable low amplitude fluctuation. It is periodically produced due to the passage of a vortex dislocation. Away from the central region the signals respond to the passage of a von Kármán vortex row.

theory. Results have shown that some basic properties of the wake-type shear flow with the vortex dislocation, including flow patterns, two incommensurable frequencies, and their beat frequency, can be well described by the lowdimensional dynamical systems. Now, the flow in the present work is considered as a typical case to investigate the influence of various nonuniform inflows on vortex dislocation and wake development by DNS approach.

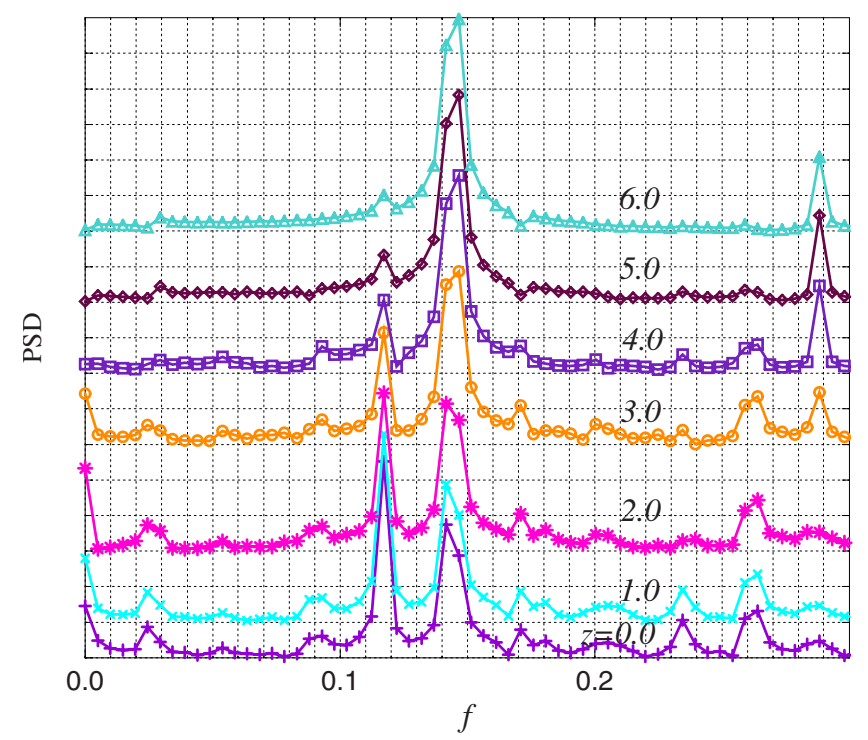

FIG. 19. (Color online) The spectra of vertical velocity component $v$. There are two incommensurable frequencies of $f_{1}=0.141$ and $f_{2}=0.117$ in the region of $z \in(0,3)$, while only one single peak $f_{1}=0.141$ occurs at $z \geq 4$. The "beat" frequency, $f_{1}-f_{2}$, at 0.0244 can be found. It shows the periodic appearance of spotlike vortex dislocation. Besides there are harmonic peaks in the spectra. 


\section{Spanwise sinusoidal wavy disturbance}

For the case of spanwise sinusoidal wavy disturbance two different degrees of waviness, a lower wave steepness $A / \lambda=0.01$ and the mild higher one $A / \lambda=0.06$, are considered in computations with parameters $n=3$ and $L=30$ in Eq. (4). These two input waviness conditions cause two distinct types of wake behavior. For the case with lower waviness, the weaker spanwise disturbance only makes Kármán vortex shedding a slight wavy deformation with a small amplitude. The wavelength of the deformed spanwise vortex tube is approximately equal to that of the imposed disturbance. The weaker streamwise and vertical vorticity are produced close to the region of inflection points of the wavy spanwise vortex. However, with the wave steepness increasing, as $A / \lambda$ $=0.06$, wake behavior displays strong unsteadiness. With increasing time, the strong wavy disturbance first causes systematic vortex dislocations along the span then results in the stabilization of the near wake to a time-independent steady state shear flow. In the early stages of flow evolution, spanwise nonuniform development of instability of the upstream flow leads to three-dimensional undulated modes of vortex shedding. Vortex shedding occurs earlier at the valley of the wavy disturbance than at the peak, resulting in a strong undulated spanwise vortex tube. In this result, there are significant phase differences between shedding cells. Vortex dislocation grows rapidly close to the region of inflection points of the spanwise wavy vortex tubes, where the rate of phase variation is at a maximum. Spanwise vortex splitting occurs there, producing noticeable streamwise vorticity and a vertical one. The overall vortex dislocation flow is shown in Fig. 20(a), where the dislocation structures are identified by the $\lambda_{2}$-definition. The vortex dislocations are characterized by main spanwise vortex tube splitting and breaking, as clearly shown in the topology of vortex structures in the figure. The frequencies of vortex shedding are calculated and are nearly the same at different spanwise positions. The mechanism responsible for the generation of vortex dislocation in the present case is the same as that of the creation of chainlike undulated vortex dislocation, caused by a local spanwise disturbance in Sec. III A. However, the difference is that in this case the vortex dislocation successively appears along the span. It is produced once each a wavelength of the imposed wavy disturbance along the span. Thus, as shown in Fig. 20(a), there are three dislocation structures across the span for each undulated spanwise vortex to form systematic spanwise periodic modes of vortex dislocations. Time-dependent distributions of vorticity components of streamwise and vertical directions are calculated and shown in Figs. 21(a) and 21(b), which are converted from the split spanwise vortex tubes. Of particular interest is the unsteadiness of the wake behavior. With the increase in time, the growth of instability of the upstream flow is found to move downstream gradually. Corresponding vortex shedding and the spanwise periodic vortex dislocation are produced downstream too, as shown in Figs. 20(b) and 20(c). At the same time, streamwise vorticity is created downstream, while the vertical component of vorticity is distributed over the whole near wake. Time sequences of these variations are given in Figs. 20 and 21. It is

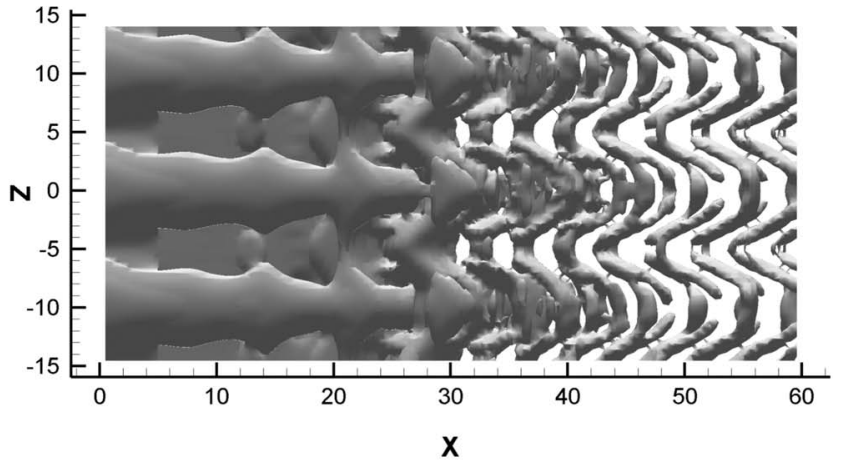

(a)

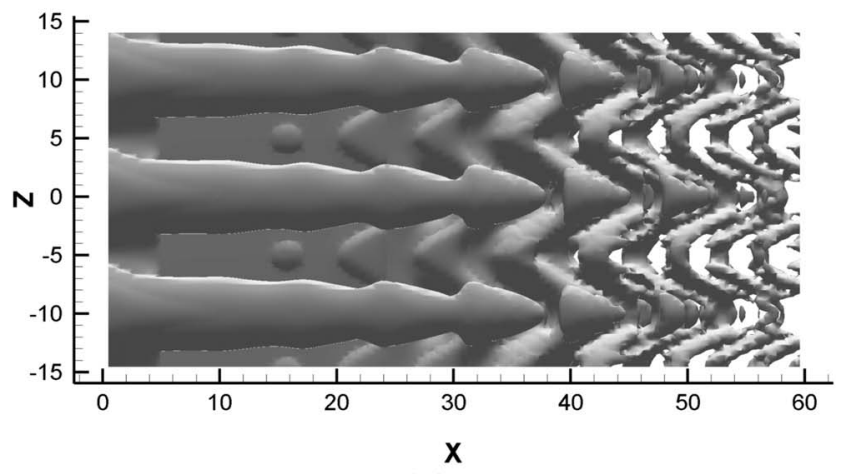

(b)

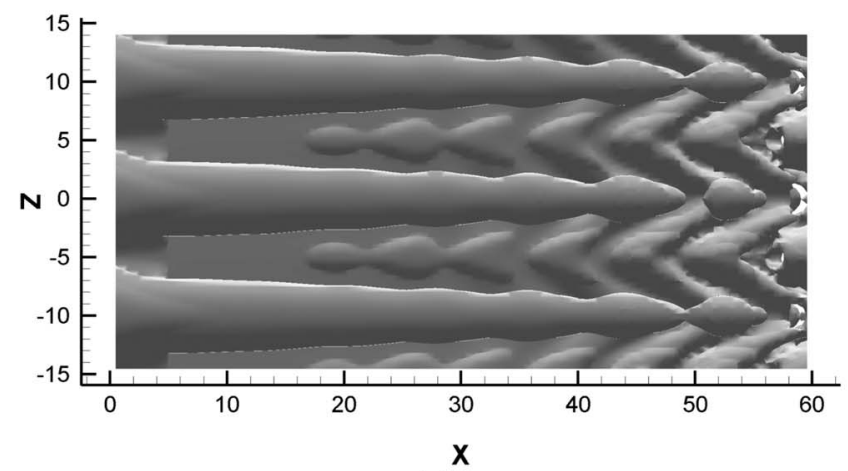

(c)

FIG. 20. Top view of time sequence of the spanwise systematic modes of vortex dislocation for $A / \lambda=0.06$ at (a) $t=80$, (b) $t=160$, and (c) $t=240$. The vortex structures are visualized by $\lambda_{2}$-definition $\left(\lambda_{2}=-0.003\right)$. The vortex dislocation is produced successively once each wavelength of the sinusoidal wave disturbance along the span and travels downstream. At $t=240$, the series dislocations move downstream almost out of the computational domain.

shown that with increasing time, vortex shedding and vortex dislocation occur further downstream and eventually move out of computational domains. Correspondingly the fluctuating velocity, induced by vortex shedding and vortex dislocation structure, decreases to zero. These features are shown in Figs. 22(a) and 22(b). The characteristics of the results suggest that the near wake has become a steady state shear flow with a vertical component of vorticity. It is hypothesized that the progressive increase in vertical vorticity in the near wake will prevent and depress the growth of spanwise vorticity. There are remarkable similarities in the phenomena and 

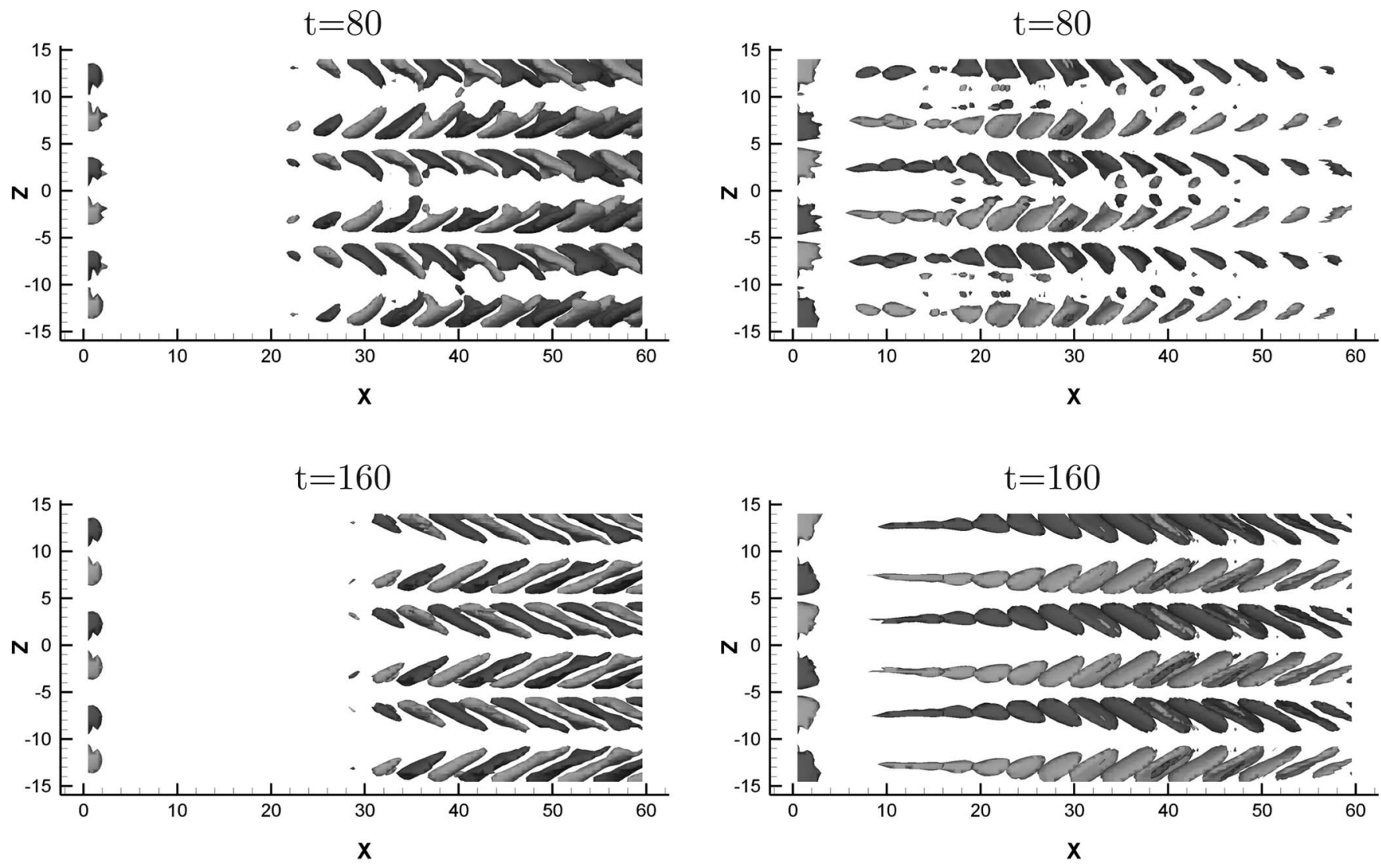

$\mathrm{t}=240$

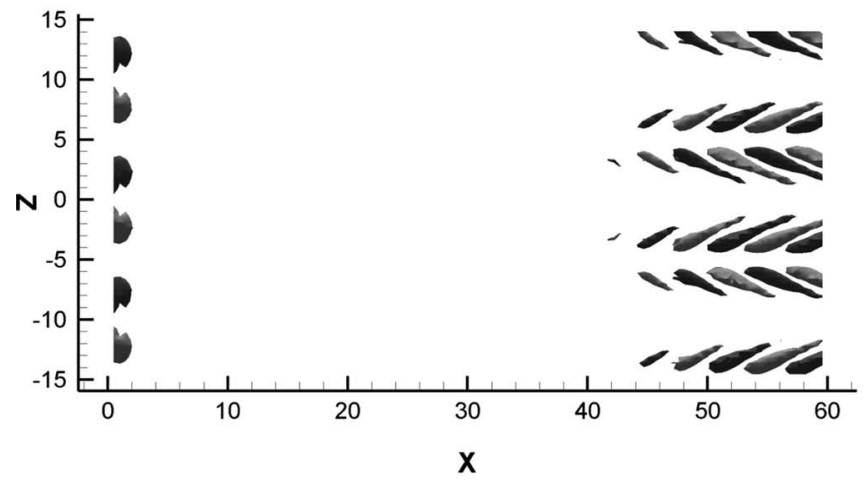

(a) $\mathrm{t}=240$

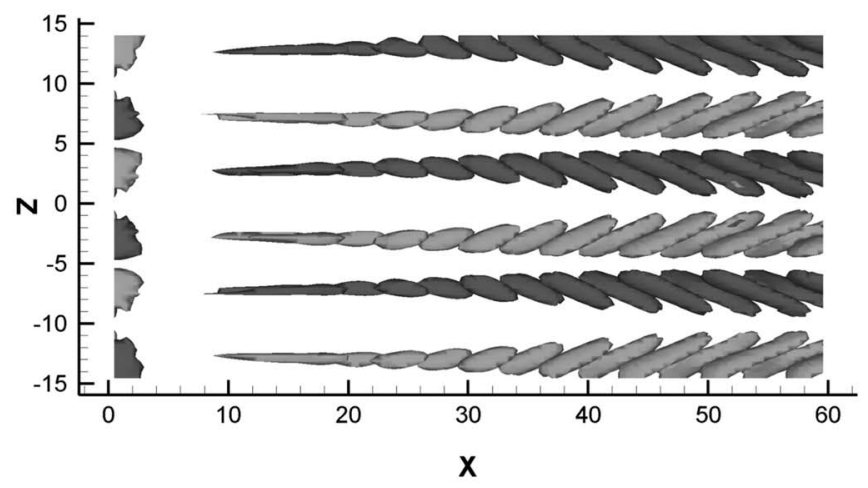

(b)

FIG. 21. The isovorticity surface of streamwise and vertical components for $A / \lambda=0.06$, (a) $\omega_{x}= \pm 0.1$ and (b) $\omega_{y}= \pm 0.1$ at $t=80,160$, and 240 . Those components of vorticity are produced by spanwise vortex splitting and reconnection. It is shown that at $t=240$ the Kármán vortex shedding almost disappears, while vertical vorticity still exists and even becomes a dominant component in the wake-type shear flow.

mechanism for suppression of the Kármán vortex shedding between the present results and those DNS results of flow around a wavy square cylinder given by Darekar and Sherwin, ${ }^{8}$ Ling and Lin, ${ }^{24}$ and Lin. ${ }^{25}$ Those recent studies of square cylinder wake have demonstrated that once the spanwise wavy disturbance is imposed on the geometry of a straight cylinder, both vertical and streamwise vortices are generated on the cylinder surface. Ling and $\mathrm{Lin}^{24}$ and $\mathrm{Lin}^{25}$ found that the spanwise wavy disturbance with a moderate waviness causes vertical vorticity to grow rapidly in the near wake and induces a local recirculating flow behind the cyl- inder. The recirculating flow prevents the spanwise vorticity development and suppresses vortex shedding.

Based on the present results together with those described in Secs. III A and III B, it can be deduced that depending on different spanwise disturbances the vortex dislocations occurred in the wake flow may play two opposite roles: It may affect the flow transition from a regular unsteady periodic vortex street to an unsteady irregular vortex flow with some turbulent behavior, as reported in cases of Secs. III A and III B, or suppress the Kármán vortex shedding and further stabilize the unsteady wake flow. 


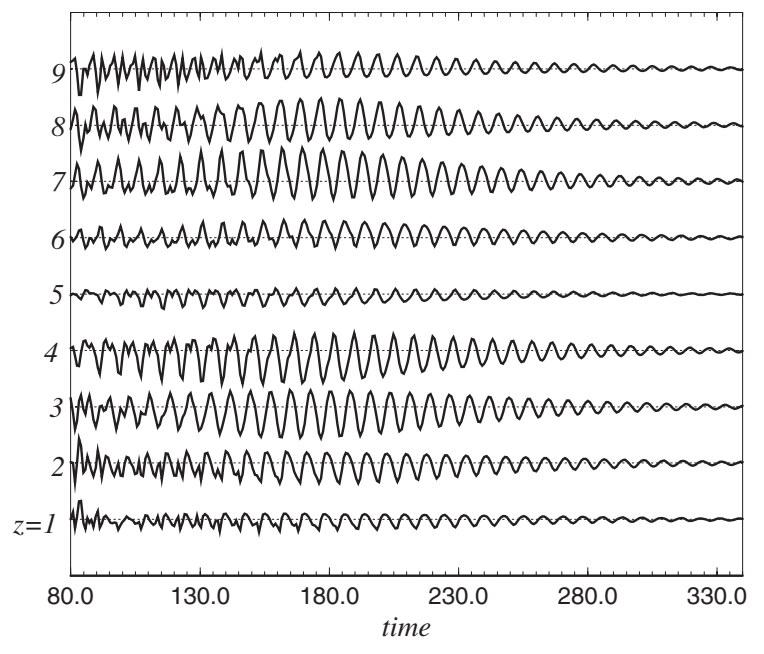

(a)

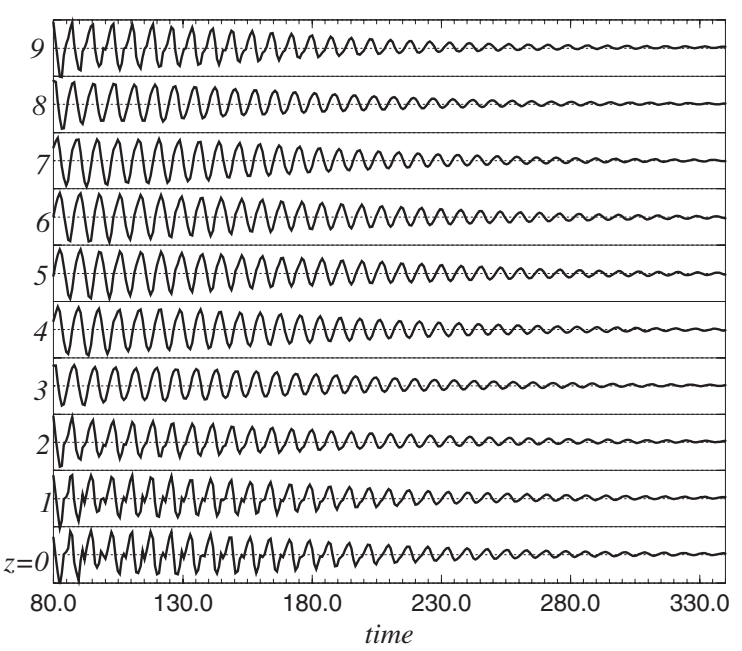

(b)

FIG. 22. Time series of fluctuation velocity (a) $v$ and (b) $w$ at $(x=40, y=1)$ for $A / \lambda=0.06$. It is clearly shown that with the increase in time the fluctuation of velocities decreases to zero that means the Kármán vortex shedding has been completely suppressed. These behaviors are consistent with the features of the variation of flow pattern and vorticity distributions in Figs. 20 and 21.

\section{CONCLUSIONS AND DISCUSSIONS}

Numerical investigations of vortex dislocation and flow transition in wake-type flow with three typical types of spanwise disturbance have been carried out by DNS approach. The principal discovery in the present work and discussions on the results may be summarized as follows.

(1) Vortex dislocations are fundamental characteristics of wake-type flow development as various spanwise disturbances are superimposed on the upstream flow.

(2) Three distinct modes of vortex dislocation generated in wake-type flows have been found, which are induced by three typical spanwise disturbances, respectively. Particularly, the local exponential decay spanwise disturbance leads to the occurrence of a systematic twisted chainlike mode of vortex dislocation, caused by phase difference between shedding cells. The stepped spanwise disturbance of upstream velocity causes a largescale spotlike mode of vortex dislocation that are produced periodically in the streamwise direction at the place with velocity discontinuity, triggered by a frequency discontinuity there. A spanwise sinusoidal wavy disturbance imposed on the inflow velocity, in the case of moderate waviness, results in a strong unsteadiness of wake behavior. First, a systematic periodic vortex dislocation occurs once each wavelength along the span to form a very complex topology of vortex dislocation structure. Then with time increasing, the Kármán vortex shedding is completely suppressed and no dislocation is found in the near wake. The near wake becomes a steady state laminar shear flow. These variations are attributed to the rapid growth of vertical component of vorticity, preventing spanwise vorticity developing.

(3) Present results have further confirmed that in wake-type flow the nature of vortex dislocations is the substantial modification of vorticity occurring on spanwise vortices from original spanwise to streamwise and vertical direc- tions, accompanied by the appearance of spanwise vortex dividing and connecting with adjacent spanwise vortices of opposite sign on the same side. These phenomena are caused by phase difference or frequency discontinuity between shedding cells.

(4) The dynamical processes related to the formation of vortex dislocation, the characteristics of vortex pattern, as well as the vortex linkages over the dislocations are illustrated in detail by examining the variations of vortex line and vorticity distribution. It has been shown that the dynamical processes are closely associated with the spanwise nonuniform development of the instability of the upstream flow, and determined by the imposed spanwise disturbances.

(5) Depending on the nonuniform spanwise disturbance, the present results have shown that the presence of vortex dislocation may play two opposite roles in wake transition. It may cause the flow to be unsteady irregular vortex flow with some turbulent behavior or suppress the Kármán vortex shedding, making wake flow steady state. Similar behavior and results can be found in the experiments of cylinder wake transition by Williamson ${ }^{1}$ and DNS results on wake vortex dynamics behind a wavy square-section cylinder by Darekar and Sherwin, ${ }^{8}$ and Ling and Lin, ${ }^{24}$ respectively.

(6) There are many close similarities and common features in the mechanism responsible for the generation of vortex dislocation and related flow phenomena between the present wake-type flow evolution and the cylinder wake transition given by previous studies.

\section{ACKNOWLEDGMENTS}

This work was supported by an Innovation Research Project of Chinese Academy of Sciences (CAS) under Grant No. KJCX2-YW-L02. Some early work of the present study 
was supported by the National Natural Science Foundation of China through Project No.10272104. The first author is grateful to Professor Jiayu Niu in Institute of Mechanics, CAS, for her joint study on the influence of nonuniform inflow on wake dynamics and for her indispensable assistance during the present work. The authors would like to thank Dr. Liming Lin for his most efficient help in typing the present manuscripts and arrangement of figures. Helpful comments and suggestions from Professor Lu Ting of the Courant Institute of Mathematical Sciences, NYU are greatly acknowledged. The authors also thank the referee of the present paper for their valuable comments and suggestions. Thanks should extend to Mr. Don Westergren, retired Program Engineer from Lockheed Martin Corp. and General Dynamics Corp. for his helpful discussion and great help in improvement of English writing.

${ }^{1}$ C. H. K. Williamson, "The natural and forced formation of spot-like vortex dislocations in the transition of a wake," J. Fluid Mech. 243, 393 (1992).

${ }^{2}$ C. H. K. Williamson, "Vortex dynamics in the cylinder wake," Annu. Rev. Fluid Mech. 28, 477 (1996)

${ }^{3}$ C. G. Lewis and M. Gharib, "An exploration of wake threedimensionalities caused by a local discontinuity in a cylinder diameter," Phys. Fluids A 4, 104 (1992).

${ }^{4} \mathrm{P}$. W. Bearman, "Challenging problems in bluff body wakes. Bluff-body wakes," in Dynamics and Instabilities, IUTAM Symposium, edited by H. Eckelmann, J. M. R. Graham, P. Huerre, and P. A. Monkewitz (SpringerVerlag, Göttingen, 1992), p. 1.

${ }^{5} \mathrm{H}$. Eisenlohr and H. Eckelemann, "Vortex splitting and its consequences in vortex street wake of cylinders at low Reynolds number," Phys. Fluids A TB1, 189 (1989).

${ }^{6}$ J. H. Gerrard, "The three-dimensional structure of the wake of a circular cylinder," J. Fluid Mech. 25, 143 (1966).

${ }^{7}$ A. Papangelou, "Vortex shedding from slender cones at low Reynolds numbers," in Bluff-Body Wakes, Dynamics and Instabilities, IUTAM Symposium, edited by H. Eckelmann, J. M. R. Graham, P. Huerre, and P. A. Monkewitz (Springer-Verlag, Göttingen, 1992), p. 361.

${ }^{8}$ R. M. Darekar and S. J. Sherwin, "Flow past a square-section cylinder with a wavy stagnation face," J. Fluid Mech. 426, 263 (2001).

${ }^{9}$ T. Dallard and F. K. Browand, "The growth of large scales at defect sites in the plane mixing layer," J. Fluid Mech. 247, 339 (1993).

${ }^{10}$ M. Braza, D. Faghani, and H. Persillson, "Successive stages and the role of natural vortex dislocation in three-dimensional wake transition," J. Fluid Mech. 439, 1 (2001).

${ }^{11}$ G. C. Ling and H. L. Zhao, "Structure of vortex dislocation in a wake-type flow," Chin. Phys. Lett. 20, 383 (2003).

${ }^{12}$ G. C. Ling and J. Y. Niu, "Numerical analysis of two distinct types of vortex dislocation in wake-type flows with different span-wise non uniformities," in MIT Conference on Computational Fluid and Solid Mechanics, edited by K. J. Bathe (Elsevier, Boston, 2005), p. 738.

${ }^{13}$ G. S. Triantafyllou and G. E. Karniadakis, "Computational reducibility of unsteady viscous flow," Phys. Fluids A 2, 653 (1990).

${ }^{14}$ G. E. Karniadakis and G. S. Triantafyllou, "Three-dimensional dynamics and transition to turbulence in the wake of bluff bodies," J. Fluid Mech. 238, 1 (1992).

${ }^{15} \mathrm{M}$. Nishioka and H. Sato, "Measurements of velocity distributions in the wake of a circular cylinder at low Reynolds numbers," J. Fluid Mech. 65, 97 (1974).

${ }^{16}$ Z. M. Xiong and G. C. Ling, "Compact finite difference-Fourier spectra method for three-dimensional incompressible Navier-Stokes equations and application to vortex dislocation," in 15th International Conference on Numerical Methods in Fluid Dynamics, edited by P. Kutler, J. Flores, and J. J. Chattot, Monterey, CA, USA (Springer, New York, 1997).

${ }^{17}$ G. C. Ling, "A numerical analysis of vortex dislocation in wake-type flow with different span-wise non uniformity," in Vortex Dominated Flows, edited by D. Blackmore, E. Krause, and C. Tung (World Scientific, Singapore, 2005), p. 157.

${ }^{18}$ G. E. Karniadakis, S. A. Israeli, and S. A. Orzag, "High-order splitting methods for the incompressible Navier-Stokes equations," J. Comput. Phys. 97, 414 (1991).

${ }^{19} \mathrm{G}$. Jin and M. Braza, "A nonreflecting outlet boundary condition for incompressible unsteady Navier-Stokes calculation,” J. Comput. Phys. 93, 107 (1993).

${ }^{20} \mathrm{H}$. Persillon and M. Braza, "Physical analysis of the transition to turbulence in the wake of a circular cylinder by three-dimensional NavierStokes simulation,” J. Fluid Mech. 365, 23 (1998).

${ }^{21}$ J. Jeong and F. Hussain, "On the identification of a vortex," J. Fluid Mech. 285, 69 (1995).

${ }^{22}$ A. Leonard, "Computing three-dimensional incompressible flows with vortex elements," Annu. Rev. Fluid Mech. 17, 523 (1985).

${ }^{23}$ H. L. Zhao and G. C. Ling, "Low-dimensional dynamical systems for a wake-type shear flow with vortex dislocations," Fluid Dyn. Res. 33, 299 (2003).

${ }^{24}$ G. C. Ling and L. M. Lin, "A note on the numerical simulations of flow past a wavy square-section cylinder," Acta Mech. Sin. 24, 101 (2008).

${ }^{25} \mathrm{~L}$. M. Lin, "Wake dynamics and forces in the flow around the squaresection cylinder with a geometric disturbance," Ph.D. thesis, Institute of Mechanics, Chinese Academy of Sciences (in Chinese), 2007. 